\title{
Design of Nonfragile State Estimator for Discrete-Time Genetic Regulatory Networks Subject to Randomly Occurring Uncertainties and Time-Varying Delays
}

\author{
Yanfeng Zhao, ${ }^{1,2}$ Jihong Shen, ${ }^{3}$ and Dongyan Chen ${ }^{4}$ \\ ${ }^{1}$ College of Automation, Harbin Engineering University, Harbin 150001, China \\ ${ }^{2}$ Graduate Department, Harbin University of Science and Technology, Harbin 150080, China \\ ${ }^{3}$ College of Science, Harbin Engineering University, Harbin 150001, China \\ ${ }^{4}$ College of Science, Harbin University of Science and Technology, Harbin 150080, China
}

Correspondence should be addressed to Yanfeng Zhao; zhaoyanfeng@hrbust.edu.cn

Received 10 March 2017; Accepted 21 May 2017; Published 2 October 2017

Academic Editor: Dimitri Volchenkov

Copyright (C) 2017 Yanfeng Zhao et al. This is an open access article distributed under the Creative Commons Attribution License, which permits unrestricted use, distribution, and reproduction in any medium, provided the original work is properly cited.

\begin{abstract}
We deal with the design problem of nonfragile state estimator for discrete-time genetic regulatory networks (GRNs) with timevarying delays and randomly occurring uncertainties. In particular, the norm-bounded uncertainties enter into the GRNs in random ways in order to reflect the characteristic of the modelling errors, and the so-called randomly occurring uncertainties are characterized by certain mutually independent random variables obeying the Bernoulli distribution. The focus of the paper is on developing a new nonfragile state estimation method to estimate the concentrations of the mRNA and the protein for considered uncertain delayed GRNs, where the randomly occurring estimator gain perturbations are allowed. By constructing a LyapunovKrasovskii functional, a delay-dependent criterion is obtained in terms of linear matrix inequalities (LMIs) by properly using the discrete-time Wirtinger-based inequality and reciprocally convex combination approach as well as the free-weighting matrix method. It is shown that the proposed method ensures that the estimation error dynamics is globally asymptotically stable and the desired estimator parameter is designed via the solutions to certain LMIs. Finally, we provide two numerical examples to illustrate the feasibility and validity of the proposed estimation results.
\end{abstract}

\section{Introduction}

Genetic regulatory networks (GRNs), which are biochemically dynamical systems describing highly complicated interactions among DNA, RNA, protein, and metabolic product in the interactive transcriptional and translational processes, have become an attractive research field in the systems biology and biomedical sciences during the past few decades. One of the main objectives in systems biology is to understand how mRNAs and proteins work collectively and interact with each other to perform the complicated biological functions (e.g., the process of transcriptions and translations). In the past few years, a great deal of attention has been devoted to the theoretical analysis and experimental investigation on GRNs. Accordingly, a large amount of important results has been reported in literature making significant contributions for understanding both static and dynamic behaviors of biological systems in detail (see [1-3] and the references therein). Among them, two main classes of models used to characterize the GRNs include discrete models such as Boolean model $[4,5]$ and continuous models such as differential equation models $[6,7]$.

When modelling GRNs, we should bear in mind the fact that GRNs models are unavoidably affected by modelling uncertainties, including parametric errors, time delays, and stochastic noises. More specifically, the time delays are unavoidable due primarily to the slow process of transcription/translation, translocation, or the finite switching speed of amplifiers. The existence of the time delays could affect the whole system performance and may lead to oscillation, divergence, and instability of the genetic networks. According to the occurrence way of time delays, they can generally be 
classified into three types: interval time-varying delays [8,9], distributed delays [10], and random time delays [11, 12]. In addition, gene regulation is an intrinsically noisy process due to intracellular and extracellular noise perturbations, which are derived from random births and deaths of individual molecules and environmental fluctuations. Accordingly, a great deal of effort has been made to deal with the analysis problem of GRNs with time-varying delays as well as stochastic noises and a growing number of approaches have been provided in the literature to examine the dynamical behaviors of the addressed GRNs [2, 3, 13]. On the other hand, the discrete-time version of GRNs receives increasing research interests due to the reason that the continuous-time GRNs are commonly discretized for computer simulation and experimental purposes $[14,15]$. In recent years, much effort has been made to tackle the synthesis problems of discrete GRNs with time delays. For example, the robust $H_{\infty}$ control problem has been investigated in [16] for discrete delayed stochastic GRNs. Moreover, the asymptotic stability problems have been investigated in [17-19] for discrete-time uncertain GRNs with time-varying delays and stochastic fluctuations, where some sufficient conditions have been presented to ensure the stability of the addressed GRNs via the linear matrix inequality approach.

In practice, for the purpose of both the drug design and disease diagnosis, it is worth mentioning that biologists hope to gain actual concentrations of gene products in GRNs. However, due to the existence of the model errors, time delays, and external disturbances, the actual values of GRNs can hardly be obtained and only partial information about the gene states is commonly available in the measurement outputs. As such, the filtering and state estimation problems for complex dynamics systems have been widely investigated; see, for example, [20-26]. To mention a few, the set-values filtering problem has been investigated in [20] for a class of GRNs with time-varying uncertainties and bounded external noise, where new filtering scheme has been given for the addressed GRNs. In [22], the state estimation problem has been studied for a class of discretetime GRNs with random delays. Moreover, the $H_{\infty}$ state estimation problem has been discussed in [24] for discrete stochastic GRNs with Markovian jumping parameters and time-varying delays. More recently, the sampling-data state estimation algorithm has been provided in [25] for a class of GRNs subject to time-varying delays. On the other hand, the parameter uncertainties may be changed in a random way with certain types and intensity due to various effects, for instance, the changing subsystem interconnections, networkinduced random failures, repairs of components and sudden environmental perturbations, and so on. Hence, it is more significant to deal with the effects from the randomly occurring parameter uncertainties onto the networked systems [2732]. In addition, as discussed in [33], a small or even tiny drift/fluctuation/error of the presented controller/estimator during the parameter implementation may result in unexpected fragility/degradation for whole system performance. In other words, there exist the deviations between the parameters of actually implemented controller/estimator and their expected values, and hence there is a need to design the robust controller/estimator with certain degree of tolerance against the possible deviations. In the past decade, the nonfragile estimation problems have gained much attention with respect to the implementation errors of proposed estimators [3436]. However, the nonfragile state estimation problem has not been fully studied yet for discrete delayed GRNs, not to mention the case where the randomly occurring parameter uncertainties are also considered. As such, the main purpose of the paper is to provide a robust state estimation method against the mentioned phenomena.

Motivated by the aforementioned analysis, in this paper, we aim to investigate the robust nonfragile state estimation problem for a class of discrete GRNs with time-varying delays and randomly occurring uncertainties. The LyapunovKrasovskii functional is chosen which utilizes more information about the time delays and a new set of conditions is added to calculate the difference of the designed LyapunovKrasovskii functional. Furthermore, as mentioned in [37], it is clearly seen that the Wirtinger-based inequality can reduce the conservatism and provide a more tighter lower delay bound of the summation terms. Therefore, we revisit the nonfragile state estimation problem for discrete GRNs with time-varying delays and randomly occurring uncertainties by using the discrete-time Wirtinger-based inequality and the reciprocally convex combination inequality. A delaydependent estimation criterion is established in terms of feasibility of a set of LMIs. The main contributions of this paper can be summarized as follows: (1) the discrete Wirtingerbased inequality is utilized for the first time to investigate the nonfragile state estimation problem for GRNs with timevarying delays and randomly occurring uncertainties, where the estimator gain perturbations occur in a random way; (2) by utilizing the discrete-time Wirtinger-based inequality and the reciprocally convex combination approach, the summation terms are addressed and more information of time delays is reflected in the proposed algorithm; and (3) more free-weighting matrices are introduced by adding to new conditions with hope to further reduce the conservativeness induced by time delays. Finally, examples and simulation are given to illustrate the effectiveness and advantages of the proposed main results.

Notations. For a matrix $A, A>0$ denotes that $A$ is a symmetric positive-definite matrix. $E[\cdot]$ stands for the mathematical expectation operator. The superscript $T$ and $(-1)$ represent the transpose and the inverse of the matrix, respectively. I represents an identity matrix with appropriate dimension. For simplicity, * represents the term that is induced by symmetry in symmetric block matrices.

\section{Model Description and Preliminaries}

In this paper, we consider the following discrete GRNs with time-varying delays, $n$ mRNA, and $n$ proteins:

$$
\begin{aligned}
M(k+1) & =A M(k)+B f(P(k-\tau(k)))+L, \\
P(k+1) & =C P(k)+D M(k-\delta(k)),
\end{aligned}
$$


where $M(k)=\left[M_{1}(k), M_{2}(k), \ldots, M_{n}(k)\right]^{T}, P(k)=\left[P_{1}(k)\right.$, $\left.P_{2}(k), \ldots, P_{n}(k)\right]^{T} \in R^{n}$ are the concentrations of mRNA and protein at instant $k$, respectively. $A=\operatorname{diag}\left\{a_{1}, a_{2}, \ldots, a_{n}\right\}$ and $C=\operatorname{diag}\left\{c_{1}, c_{2}, \ldots, c_{n}\right\}$ are real constant diagonal matrices representing the decay rates of mRNA and protein with entries $\left|a_{i}\right|<1$ and $\left|c_{i}\right|<1, D=\operatorname{diag}\left\{d_{1}, d_{2}, \ldots, d_{n}\right\}, d_{i}$ is the translation rate, and $B=\left(b_{i j}\right)_{n \times n}$ is the coupling matrix of the genetic networks. $L=\left[l_{1}, l_{2}, \ldots, l_{n}\right]^{T}$ stands for the basal rates of degradation. $\tau(k)>0$ and $\delta(k)>0$ are the timevarying delays denoting the feedback regulation delay and the translation delay satisfying $0<\tau_{m} \leq \tau(k) \leq \tau_{M}$ and $0<$ $\delta_{m} \leq \delta(k) \leq \delta_{M}$. In addition, the nonlinear function $f(k)=$ $\left[f_{1}(k), f_{2}(k), \ldots, f_{n}(k)\right]^{T}$ represents the feedback regulation of the protein on the transcription. It is a monotonic function in the Hill form; that is, $f_{j}(x)=\left(x / \beta_{j}\right)^{H_{j}} /\left(1+\left(x / \beta_{j}\right)^{H_{j}}\right)$, $x \in R$, where $H_{j}$ is the Hill coefficient and $\beta_{j}$ is a positive constant.

Let $\left[M^{*^{T}}, P^{*^{T}}\right]=\left[M_{1}^{*}, M_{2}^{*}, \ldots, M_{n}^{*}, P_{1}^{*}, P_{2}^{*}, \ldots, P_{n}^{*}\right]$ be an equilibrium point of system (1). Then, it is easy to obtain that

$$
\begin{aligned}
M^{*} & =A M^{*}+B f\left(P^{*}\right)+L, \\
P^{*} & =C P^{*}+D M^{*} .
\end{aligned}
$$

Subsequently, we can shift the equilibrium point $\left[M^{*^{T}}, P^{*^{T}}\right]$ of system (1) to the origin point via the transformations $x(k)=M(k)-M^{*}$ and $y(k)=P(k)-P^{*}$. Then, system (1) can be converted into the following form:

$$
\begin{aligned}
& x(k+1)=A x(k)+B g(y(k-\tau(k))), \\
& y(k+1)=C y(k)+D x(k-\delta(k)) .
\end{aligned}
$$

with $g(y(k))=f\left(y(k)+P^{*}\right)-f\left(P^{*}\right)$.

As mentioned above, the GRNs are often the large-scale networks and it should be noted that system parameter uncertainties may be subject to random changes in real circumstances due to some factors such as repairs of components and sudden environmental disturbances. Thus, they may occur in a probabilistic way with various types and intensities. By considering the randomly occurring uncertainties, system (3) becomes

$$
\begin{aligned}
x(k+1)= & \left(A+\alpha_{1}(k) \Delta A(k)\right) x(k) \\
& +\left(B+\beta_{2}(k) \Delta B(k)\right) g(y(k-\tau(k))), \\
y(k+1)= & \left(C+\beta_{1}(k) \Delta C(k)\right) y(k) \\
& +\left(D+\alpha_{2}(k) \Delta D(k)\right) x(k-\delta(k)),
\end{aligned}
$$

where $\Delta A(k), \Delta B(k), \Delta C(k)$, and $\Delta D(k)$ denote the parameter uncertainties satisfying the following condition:

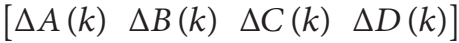

$$
\begin{aligned}
& =E_{1} F(k)\left[\begin{array}{llll}
M_{1} & M_{2} & M_{3} & M_{4}
\end{array}\right],
\end{aligned}
$$

where $E_{1}, M_{1}, M_{2}, M_{3}$, and $M_{4}$ are known constant matrices and $F(k)$ is an unknown time-varying matrix satisfying $F^{T}(k) F(k) \leq I$. The stochastic variables $\alpha_{i}(k)$ and $\beta_{i}(k)(i=$ $1,2)$ are used to characterize the randomly occurring uncertainties and are mutually independent Bernoulli-distributed white noise sequences satisfying

$$
\begin{aligned}
& \operatorname{Prob}\left\{\alpha_{i}(k)=1\right\}=\alpha_{i}, \\
& \operatorname{Prob}\left\{\alpha_{i}(k)=0\right\}=1-\alpha_{i}, \\
& \operatorname{Prob}\left\{\beta_{i}(k)=1\right\}=\beta_{i}, \\
& \operatorname{Prob}\left\{\beta_{i}(k)=0\right\}=1-\beta_{i},
\end{aligned}
$$

where $\alpha_{i}$ and $\beta_{i} \in[0,1](i=1,2)$ are known constants.

The state components of the GRNs are usually not completely accessible. Consequently, it is necessary to make use of the available output information and design a state estimator to estimate the state vector of the addressed GRNs. For this case, assume that the measurement outputs are given as follows:

$$
\begin{aligned}
& z_{x}(k)=W_{1} x(k), \\
& z_{y}(k)=W_{2} y(k),
\end{aligned}
$$

where $z_{x}(k)$ and $z_{y}(k)$ are the measurements of model (4); $W_{1}$ and $W_{2}$ are the known matrices.

The main objective of this paper is to estimate the concentrations of mRNA and protein in (4) from the available network outputs in (7). In the sequel, we design the following nonfragile state estimator:

$$
\begin{aligned}
\widehat{x}(k+1) & \\
= & A \hat{x}(k)+B g(\hat{y}(k-\tau(k))) \\
& +\left(K_{1}+\gamma_{1}(k) \Delta K_{1}(k)\right)\left[z_{x}(k)-W_{1} \hat{x}(k)\right], \\
\hat{y}(k+1) & \\
= & C \hat{y}(k)+D \hat{x}(k-\delta(k)) \\
& +\left(K_{2}+\gamma_{2}(k) \Delta K_{2}(k)\right)\left[z_{y}(k)-W_{2} \hat{y}(k)\right],
\end{aligned}
$$

where $\hat{x}(k), \hat{y}(k)$ are the estimations of $x(k)$ and $y(k)$, and the nonfragile estimator gain matrices $K_{1}, K_{2}$ are to be determined. The real-valued matrices $\Delta K_{1}(k)$ and $\Delta K_{2}(k)$ represent possible estimator gain fluctuations. It is assumed that $\Delta K_{1}(k)$ and $\Delta K_{2}(k)$ have the following structure:

$$
\left[\Delta K_{1}(k) \Delta K_{2}(k)\right]=E_{2} F(k)\left[\begin{array}{ll}
M_{5} & M_{6}
\end{array}\right],
$$

where $E_{2}, M_{5}$, and $M_{6}$ are the known constant matrices and $F(k)$ is the unknown time-varying matrix satisfying $F^{T}(k) F(k) \leq I$. The stochastic variables $\gamma_{i}(k)(i=1,2)$ 
represent the perturbations in the estimator gain matrices and are mutually independent Bernoulli-distributed white noise sequences taking on values of 0 or 1 with

$$
\begin{aligned}
& \operatorname{Prob}\left\{\gamma_{i}(k)=1\right\}=\gamma_{i}, \\
& \operatorname{Prob}\left\{\gamma_{i}(k)=0\right\}=1-\gamma_{i},
\end{aligned}
$$

where $\gamma_{i} \in[0,1](i=1,2)$ are known constants.

Letting the estimation error be $\tilde{x}(k)=x(k)-\widehat{x}(k)$ and $\tilde{y}(k)=y(k)-\widehat{y}(k)$, the estimation error dynamics can be described as follows:

$$
\begin{aligned}
\tilde{x}(k+1)= & \left(A-K_{1} W_{1}\right) \tilde{x}(k)+B g(\tilde{y}(k-\tau(k))) \\
& +\alpha_{1}(k) \Delta A(k) x(k) \\
& -\gamma_{1}(k) \Delta K_{1}(k) W_{1} \tilde{x}(k) \\
& +\beta_{2}(k) \Delta B(k) g(y(k-\tau(k))) \\
\tilde{y}(k+1)= & \left(C-K_{2} W_{2}\right) \tilde{y}(k)+D \tilde{x}(k-\delta(k)) \\
& +\beta_{1}(k) \Delta C(k) y(k) \\
& -\gamma_{2}(k) \Delta K_{2}(k) W_{2} \tilde{y}(k) \\
& +\alpha_{2}(k) \Delta D(k) x(k-\delta(k))
\end{aligned}
$$

where $g(\tilde{y}(k-\tau(k)))=g(y(k-\tau(k)))-g(\widehat{y}(k-\tau(k)))$. Initial conditions for the uncertain GRNs $(4)$ and state estimator system (8) are assumed to be $(x(k), y(k))=(\phi(k), \psi(k))$ and $(\widehat{x}(k), \widehat{y}(k))=(\widehat{\phi}(k), \widehat{\psi}(k))$ on $[-\tau, 0]$, where $\tau=$ $\max \left\{\tau_{M}, \delta_{M}\right\}$.

To facilitate further derivations, set

$$
\begin{aligned}
& e_{x}(k)=\left[\begin{array}{c}
x(k) \\
\tilde{x}(k)
\end{array}\right], \\
& e_{y}(k)=\left[\begin{array}{c}
y(k) \\
\tilde{y}(k)
\end{array}\right] .
\end{aligned}
$$

Combining (4) with (11), we obtain the augmented estimation error dynamics:

$$
\begin{aligned}
e_{x}(k+1)= & \bar{A}_{1} e_{x}(k)+\bar{B}_{1} g\left(e_{y}(k-\tau(k))\right) \\
& +\bar{E}_{1} \varrho_{1}(k)+\bar{E}_{2} \varrho_{2}(k), \\
e_{y}(k+1)= & \bar{C}_{1} e_{y}(k)+\bar{D}_{1} e_{x}(k-\delta(k))+\bar{E}_{1} \varrho_{3}(k) \\
& +\bar{E}_{2} \varrho_{4}(k), \\
\varrho_{i}(k)= & \bar{F}(k) \varsigma_{i}(k), \quad i=1,2,3,4,
\end{aligned}
$$

$$
\begin{aligned}
\varsigma_{1}(k)= & \alpha_{1}(k) \bar{M}_{1} e_{x}(k) \\
& +\beta_{2}(k) \bar{M}_{2} g\left(e_{y}(k-\tau(k))\right), \\
\varsigma_{2}(k)= & \gamma_{1}(k) \bar{M}_{5} e_{x}(k), \\
\varsigma_{3}(k)= & \beta_{1}(k) \bar{M}_{3} e_{y}(k) \\
& +\alpha_{2}(k) \bar{M}_{4} e_{x}(k-\delta(k)), \\
\varsigma_{4}(k)= & \gamma_{2}(k) \bar{M}_{6} e_{y}(k),
\end{aligned}
$$

where

$$
\begin{aligned}
& \bar{A}_{1}=\left[\begin{array}{cc}
A & 0 \\
0 & A-K_{1} W_{1}
\end{array}\right], \\
& \bar{C}_{1}=\left[\begin{array}{cc}
C & 0 \\
0 & C-K_{2} W_{2}
\end{array}\right], \\
& \bar{B}_{1}=\left[\begin{array}{ll}
B & 0 \\
0 & B
\end{array}\right], \\
& \bar{D}_{1}=\left[\begin{array}{cc}
D & 0 \\
0 & D
\end{array}\right], \\
& \bar{E}_{1}=\left[\begin{array}{cc}
E_{1} & 0 \\
0 & E_{1}
\end{array}\right], \\
& \bar{E}_{2}=\left[\begin{array}{cc}
E_{2} & 0 \\
0 & E_{2}
\end{array}\right],
\end{aligned}
$$$$
\bar{F}(k)=\left[\begin{array}{cc}
F(k) & 0 \\
0 & F(k)
\end{array}\right],
$$$$
\bar{M}_{1}=\left[\begin{array}{ll}
M_{1} & 0 \\
M_{1} & 0
\end{array}\right],
$$$$
\bar{M}_{2}=\left[\begin{array}{ll}
M_{2} & 0 \\
M_{2} & 0
\end{array}\right] \text {, }
$$$$
\bar{M}_{3}=\left[\begin{array}{ll}
M_{3} & 0 \\
M_{3} & 0
\end{array}\right],
$$$$
\bar{M}_{4}=\left[\begin{array}{ll}
M_{4} & 0 \\
M_{4} & 0
\end{array}\right],
$$$$
\bar{M}_{5}=\left[\begin{array}{cc}
0 & 0 \\
0 & -M_{5} W_{1}
\end{array}\right] \text {, }
$$$$
\bar{M}_{6}=\left[\begin{array}{cc}
0 & 0 \\
0 & -M_{6} W_{2}
\end{array}\right] \text {. }
$$ 
Assumption 1. The nonlinear function $g_{i}(\cdot)(i=1,2, \ldots, n)$ is a monotonically increasing function and satisfies the following condition:

$$
0 \leq \frac{g_{i}\left(x_{i}\right)}{x_{i}} \leq l_{i}, \quad x_{i} \neq 0, g_{i}(0)=0,
$$

which is equivalent to

$$
g^{T}(x)(g(x)-L x) \leq 0,
$$

where $L=\operatorname{diag}\left\{l_{1}, l_{2}, \ldots, l_{n}\right\}>0$ and $l_{i}(i=1,2, \ldots, n)$ are known constants.

Definition 2. System (8) is said to be a robust asymptotic state estimator of the GRNs (4) if estimation error system (13) is globally robustly asymptotically stable in the mean square; that is,

$$
\lim _{k \rightarrow+\infty} E\left\{\|\tilde{x}(k)\|^{2}+\|\tilde{y}(k)\|^{2}\right\}=0
$$

To end of this section, we introduce the following lemmas which will be frequently used in the subsequent developments.

Lemma 3 ([38] (Schur complement)). Given constant matrices $S_{11}, S_{12}$, and $S_{22}$ with appropriate dimensions, where $S_{11}=$ $S_{11}^{T}$ and $S_{22}=S_{22}^{T}<0$, then $S_{11}-S_{12} S_{22}^{-1} S_{12}^{T}<0$ if and only if

$$
S=\left[\begin{array}{ll}
S_{11} & S_{12} \\
S_{12}^{T} & S_{22}
\end{array}\right]<0
$$

Lemma 4 ([37] (discrete-time Wirtinger-based inequality)). For a given positive-definite matrix $R$ and three nonnegative integers $a, b, k$ satisfying $a \leq b \leq k$, denote

$$
\begin{aligned}
& \chi(k, a, b) \\
& = \begin{cases}\frac{1}{b-a}\left[\left(2 \sum_{j=k-b}^{k-a-1} x(j)\right)+x(k-a)-x(k-b)\right], & (a<b) \\
2 x(k-a), & (a=b) .\end{cases}
\end{aligned}
$$

Then, one has

$$
\begin{gathered}
-(b-a) \sum_{j=k-b}^{k-a-1} \eta^{T}(i) R \eta(i) \\
\leq-\left[\begin{array}{c}
\Omega_{0} \\
\Omega_{1}
\end{array}\right]^{T}\left[\begin{array}{cc}
R & 0 \\
0 & 3 R
\end{array}\right]\left[\begin{array}{l}
\Omega_{0} \\
\Omega_{1}
\end{array}\right],
\end{gathered}
$$

where

$$
\begin{aligned}
\eta(k) & =x(k+1)-x(k), \\
\Omega_{0} & =x(k-a)-x(k-b), \\
\Omega_{1} & =x(k-a)+x(k-b)-\chi(k, a, b) .
\end{aligned}
$$

Lemma 5 (see [39]). For given positive integers $n, m$, a scalar $\alpha \in(0,1)$, an $n \times n$-matrix $R>0$, and two $n \times m$-matrices $S_{1}, S_{2}$, for all vector $\xi \in R^{m}$, the function $\Theta(\alpha, R)$ is given by

$$
\Theta(\alpha, R)=\frac{1}{\alpha} \xi^{T} S_{1}^{T} R S_{1} \xi+\frac{1}{1-\alpha} \xi^{T} S_{2}^{T} R S_{2} \xi
$$

If there is a matrix $X \in R^{n \times n}$ such that $\left[\begin{array}{ll}R & X \\ * & R\end{array}\right]>0$, then the following inequality holds:

$$
\min \Theta(\alpha, R) \geq\left[\begin{array}{l}
S_{1} \xi \\
S_{2} \xi
\end{array}\right]^{T}\left[\begin{array}{ll}
R & X \\
* & R
\end{array}\right]\left[\begin{array}{l}
S_{1} \xi \\
S_{2} \xi
\end{array}\right]
$$

\section{Main Results}

In this section, we first consider that there are no parameter uncertainties in GRNs (4). Also, the estimator can be selected as (8); then augmented error system (13) can be rewritten as

$$
\begin{aligned}
e_{x}(k+1)= & \bar{A}_{1} e_{x}(k)+\bar{B}_{1} g\left(e_{y}(k-\tau(k))\right) \\
& +\bar{E}_{2} \varrho_{2}(k), \\
e_{y}(k+1)= & \bar{C}_{1} e_{y}(k)+\bar{D}_{1} e_{x}(k-\delta(k))+\bar{E}_{2} \varrho_{4}(k), \\
\varrho_{i}(k)= & \bar{F}(k) \varsigma_{i}(k), \quad i=2,4, \\
\varsigma_{2}(k)= & \gamma_{1}(k) \bar{M}_{5} e_{x}(k), \\
\varsigma_{4}(k)= & \gamma_{2}(k) \bar{M}_{6} e_{y}(k) .
\end{aligned}
$$

Our main aim is to design the nonfragile state estimator to guarantee that estimation error system (24) is globally asymptotically stable. In other words, by designing a proper Lyapunov-Krasovskii functional, together with the discretetime Wirtinger-based inequality, reciprocally convex combination approach, and the free-weighting matrices method, we are interested in looking for the estimator gain matrices $K_{1}$ and $K_{2}$ such that the error dynamics governed by (24) is globally asymptotically stable. Further, the result is extended to handle the nonfragile state estimation problem for GRNs (4) with randomly occurring uncertainties.

Theorem 6. For given positive scalars $\delta_{m}, \delta_{M}, \tau_{m}$, and $\tau_{M}$, estimation error system (24) is globally asymptotically stable, if there exist scalars $\varepsilon_{i}>0(i=2,4)$, matrices $P_{1}>0, P_{2}>0$, $R>0, Z_{j}>0(j=1,2,3,4)$, and $Q_{l}>0(l=1,2, \ldots, 6)$, positive diagonal matrices $\Lambda_{1}, \Lambda_{2}$, and $\Lambda_{3}$, matrices $Y_{i}=$ $\left[\begin{array}{cc}Y_{i 1} & Y_{i 2} \\ * & Y_{i 3}\end{array}\right](i=1,2)$, and block diagonal matrices $N_{1}, N_{2}, N_{3}$, $N_{4}, X_{1}$, and $X_{3}$, such that the following LMIs hold:

$$
\Sigma=\left[\begin{array}{ccc}
\Xi^{0} & \varepsilon_{2} \Psi_{2}^{T} & \varepsilon_{4} \Psi_{4}^{T} \\
* & -\varepsilon_{2} I_{2 n} & 0 \\
* & * & -\varepsilon_{4} I_{2 n}
\end{array}\right]<0,
$$


where

$$
\begin{aligned}
& \Psi_{2}=\left[\begin{array}{ll}
\gamma_{1} \bar{M}_{5} & 0_{n \times 19 n}
\end{array}\right], \\
& \Psi_{4}=\left[\begin{array}{lll}
0_{n \times 4 n} & \gamma_{2} \bar{M}_{6} & 0_{n \times 15 n}
\end{array}\right], \\
& \Xi^{0}=\sum_{i=1}^{9} \Xi_{i}, \\
& \Xi_{1}=2 e_{1} P_{1} e_{9}^{T}+e_{9} P_{1} e_{9}^{T}+2 e_{5} P_{2} e_{10}^{T}+e_{10} P_{2} e_{10}^{T}, \\
& \Xi_{2}=e_{1}\left(Q_{1}+\left(1+\delta_{1}\right) Q_{2}+Q_{3}\right) e_{1}^{T}-e_{3} Q_{1} e_{3}^{T} \\
& -e_{2} Q_{2} e_{2}^{T}-e_{4} Q_{3} e_{4}^{T}, \\
& \Xi_{3}=e_{5}\left(Q_{4}+\left(1+\tau_{1}\right) Q_{5}+Q_{6}\right) e_{5}^{T}-e_{7} Q_{4} e_{7}^{T} \\
& -e_{6} Q_{5} e_{6}^{T}-e_{8} Q_{6} e_{8}^{T}, \\
& \Xi_{4}=e_{11}\left(1+\tau_{1}\right) R e_{11}^{T}-e_{12} R e_{12}^{T}, \\
& \Xi_{5}=e_{9}\left(\delta_{1} Z_{1}+\delta_{m} Z_{2}\right) e_{9}^{T}+e_{10}\left(\tau_{1} Z_{3}+\tau_{m} Z_{4}\right) e_{10}^{T} \\
& -\frac{1}{\delta_{m}} \rho_{1} \widetilde{Z}_{2} \rho_{1}^{T}-\frac{1}{\delta_{1}} \rho_{7} \Theta_{1} \rho_{7}^{T}-\frac{1}{\tau_{m}} \rho_{4} \widetilde{Z}_{4} \rho_{4}^{T} \\
& -\frac{1}{\tau_{1}} \rho_{8} \Theta_{2} \rho_{8}^{T} \text {, } \\
& \Xi_{6}=2 e_{5} L \Lambda_{1} e_{11}^{T}-2 e_{11} \Lambda_{1} e_{11}^{T}+2 e_{6} L \Lambda_{2} e_{12}^{T} \\
& -2 e_{12} \Lambda_{2} e_{12}^{T}+2\left(e_{5}-e_{6}\right) L \Lambda_{3}\left(e_{11}-e_{12}\right)^{T} \\
& -2\left(e_{11}-e_{12}\right) \Lambda_{3}\left(e_{11}-e_{12}\right)^{T}, \\
& \Xi_{7}=-e_{19} \varepsilon_{2} I_{2 n} e_{19}^{T}-e_{20} \varepsilon_{4} I_{2 n} e_{20}^{T}, \\
& \Xi_{8}=2 e_{9} N_{1} \bar{B}_{1} e_{12}^{T}+2 e_{9} N_{1} \bar{E}_{2} e_{19}^{T}-2 e_{9} N_{1} e_{9}^{T} \\
& +2 e_{1} N_{2} \bar{B}_{1} e_{12}^{T}+2 e_{1} N_{2} \bar{E}_{2} e_{19}^{T}-2 e_{1} N_{2} e_{9}^{T} \\
& +2 e_{10} N_{3} \bar{D}_{1} e_{2}^{T}+2 e_{10} N_{3} \bar{E}_{2} e_{20}^{T}-2 e_{10} N_{3} e_{10}^{T} \\
& +2 e_{5} N_{4} \bar{D}_{1} e_{2}^{T}+2 e_{5} N_{4} \bar{E}_{2} e_{20}^{T}-2 e_{5} N_{4} e_{10}^{T} \text {, } \\
& \Xi_{9}=2 e_{9}\left(N_{1} \widetilde{A}_{1}-X_{1} \bar{W}_{1}\right) e_{1}^{T} \\
& +2 e_{1}\left(N_{2} \widetilde{A}_{1}-X_{1} \bar{W}_{1}\right) e_{1}^{T} \\
& +2 e_{10}\left(N_{3} \widetilde{C}_{1}-X_{3} \bar{W}_{2}\right) e_{5}^{T} \\
& +2 e_{5}\left(N_{4} \widetilde{C}_{1}-X_{3} \bar{W}_{2}\right) e_{5}^{T} \text {, } \\
& e_{i}=\left[\begin{array}{lll}
0_{2 n \times(i-1) 2 n} & I_{2 n \times 2 n} & 0_{2 n \times(20-i) 2 n}
\end{array}\right]^{T}, \\
& i=1,2, \ldots, 20 \text {, }
\end{aligned}
$$

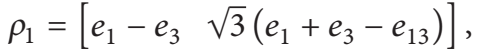

$$
\begin{aligned}
\rho_{2} & =\left[\begin{array}{ll}
e_{2}-e_{4} & \sqrt{3}\left(e_{2}+e_{4}-e_{14}\right)
\end{array}\right], \\
\rho_{3} & =\left[\begin{array}{ll}
e_{3}-e_{2} & \sqrt{3}\left(e_{3}+e_{2}-e_{15}\right)
\end{array}\right], \\
\rho_{4} & =\left[\begin{array}{ll}
e_{5}-e_{7} & \sqrt{3}\left(e_{5}+e_{7}-e_{16}\right)
\end{array}\right], \\
\rho_{5} & =\left[\begin{array}{ll}
e_{6}-e_{8} & \sqrt{3}\left(e_{6}+e_{8}-e_{17}\right)
\end{array}\right], \\
\rho_{6} & =\left[\begin{array}{ll}
e_{7}-e_{6} & \sqrt{3}\left(e_{7}+e_{6}-e_{18}\right)
\end{array}\right], \\
\rho_{7} & =\left[\begin{array}{ll}
\rho_{2} & \rho_{3}
\end{array}\right], \\
\rho_{8} & =\left[\begin{array}{ll}
\rho_{5} & \rho_{6}
\end{array}\right], \\
\widetilde{Z}_{i} & =\left[\begin{array}{cc}
Z_{i} & 0 \\
0 & Z_{i}
\end{array}\right], \quad(i=1,2,3,4), \\
\widetilde{C}_{1} & =\left[\begin{array}{ll}
C-I & 0 \\
\Theta_{1} & =\left[\begin{array}{ll}
0 & 0 \\
0 & -W_{2}
\end{array}\right] . \\
\widetilde{Z}_{1} & Y_{1} \\
* & \widetilde{Z}_{1}
\end{array}\right], \\
\widetilde{A}_{1} & =\left[\begin{array}{ll}
A-I & 0 \\
\Theta_{2} & =\left[\begin{array}{ll}
0 & 0 \\
\widetilde{Z}_{3} & Y_{2} \\
* & \widetilde{Z}_{3}
\end{array}\right],
\end{array}\right],
\end{aligned}
$$

And the remaining terms are zero. Then, the estimator gain matrices are determined by

$$
\begin{aligned}
& K_{1}=N_{12}^{-1} X_{1}, \\
& K_{2}=N_{32}^{-1} X_{3} .
\end{aligned}
$$

Proof. Set

$$
\begin{aligned}
\eta_{1}(k) & =e_{x}(k+1)-e_{x}(k), \\
\eta_{2}(k) & =e_{y}(k+1)-e_{y}(k), \\
\delta_{1} & =\delta_{M}-\delta_{m}, \\
\tau_{1} & =\tau_{M}-\tau_{m} .
\end{aligned}
$$

We construct the following Lyapunov-Krasovskii functional for discrete-time GRNs (24):

$$
V(k)=\sum_{i=1}^{5} V_{i}(k),
$$


where

$$
\begin{aligned}
& V_{1}(k)=e_{x}^{T}(k) P_{1} e_{x}(k)+e_{y}^{T}(k) P_{2} e_{y}(k), \\
& V_{2}(k)=\sum_{i=k-\delta_{m}}^{k-1} e_{x}^{T}(i) Q_{1} e_{x}(i)+\sum_{i=k-\delta_{M}}^{k-1} e_{x}^{T}(i) Q_{3} e_{x}(i) \\
& +\sum_{i=k-\delta(k)}^{k-1} e_{x}^{T}(i) Q_{2} e_{x}(i) \\
& +\sum_{j=-\delta_{M}+1}^{-\delta_{m}} \sum_{i=k+j}^{k-1} e_{x}^{T}(i) Q_{2} e_{x}(i), \\
& V_{3}(k)=\sum_{i=k-\tau_{m}}^{k-1} e_{y}{ }^{T}(i) Q_{4} e_{y}(i)+\sum_{i=k-\tau_{M}}^{k-1} e_{y}{ }^{T}(i) Q_{6} e_{y}(i) \\
& +\sum_{i=k-\tau(k)}^{k-1} e_{y}^{T}(i) Q_{5} e_{y}(i) \\
& +\sum_{j=-\tau_{M}+1}^{-\tau_{m}} \sum_{i=k+j}^{k-1} e_{y}^{T}(i) Q_{5} e_{y}(i), \\
& V_{4}(k)=\sum_{i=k-\tau(k)}^{k-1} g^{T}\left(e_{y}(i)\right) R g\left(e_{y}(i)\right) \\
& +\sum_{j=-\tau_{M}+1}^{-\tau_{m}} \sum_{i=k+j}^{k-1} g^{T}\left(e_{y}(i)\right) R g\left(e_{y}(i)\right), \\
& V_{5}(k)=\sum_{j=-\delta_{M}}^{-\delta_{m}-1} \sum_{i=k+j}^{k-1} \eta_{1}^{T}(i) Z_{1} \eta_{1}(i) \\
& +\sum_{j=-\delta_{m}}^{-1} \sum_{i=k+j}^{k-1} \eta_{1}^{T}(i) Z_{2} \eta_{1}(i) \\
& +\sum_{j=-\tau_{M}}^{-\tau_{m}-1} \sum_{i=k+j}^{k-1} \eta_{2}^{T}(i) Z_{3} \eta_{2}(i) \\
& +\sum_{j=-\tau_{m}}^{-1} \sum_{i=k+j}^{k-1} \eta_{2}^{T}(i) Z_{4} \eta_{2}(i)
\end{aligned}
$$

For convenience, define the following notations:

$$
\begin{aligned}
& \chi_{1}(k)=\chi\left(k, 0, \delta_{m}\right), \\
& \chi_{2}(k)=\chi\left(k, \delta(k), \delta_{M}\right), \\
& \chi_{3}(k)=\chi\left(k, \delta_{m}, \delta(k)\right), \\
& \chi_{4}(k)=\chi\left(k, 0, \tau_{m}\right), \\
& \chi_{5}(k)=\chi\left(k, \tau(k), \tau_{M}\right),
\end{aligned}
$$

$$
\begin{aligned}
& \chi_{6}(k)=\chi\left(k, \tau_{m}, \tau(k)\right), \\
& \xi^{T}(k)=\left[e_{x}^{T}(k), e_{x}^{T}(k-\delta(k)), e_{x}^{T}\left(k-\delta_{m}\right),\right. \\
& \quad e_{x}^{T}\left(k-\delta_{M}\right), e_{y}^{T}(k), e_{y}^{T}(k-\tau(k)), e_{y}^{T}\left(k-\tau_{m}\right), \\
& \quad e_{y}^{T}\left(k-\tau_{M}\right), \eta_{1}^{T}(k), \eta_{2}^{T}(k), g^{T}\left(e_{y}(k)\right), \\
& \quad g^{T}\left(e_{y}(k-\tau(k))\right), \chi_{1}^{T}(k), \chi_{2}^{T}(k), \chi_{3}^{T}(k), \chi_{4}^{T}(k), \\
& \left.\quad \chi_{5}^{T}(k), \chi_{6}^{T}(k), \varrho_{2}^{T}(k), \varrho_{4}^{T}(k)\right] .
\end{aligned}
$$

Calculate the difference of $V(k)$ by defining $\Delta V(k)=V(k+$ $1)-V(k)$ along the solutions of (24):

$$
\begin{aligned}
& \Delta V_{1}(k)=V_{1}(k+1)-V_{1}(k) \\
& =e_{x}^{T}(k+1) P_{1} e_{x}(k+1)-e_{x}^{T}(k) P_{1} e_{x}(k) \\
& +e_{y}^{T}(k+1) P_{2} e_{y}(k+1)-e_{y}^{T}(k) P_{2} e_{y}(k) \\
& =\left(\eta_{1}(k)+e_{x}(k)\right)^{T} P_{1}\left(\eta_{1}(k)+e_{x}(k)\right) \\
& -e_{x}^{T}(k) P_{1} e_{x}(k) \\
& +\left(\eta_{2}(k)+e_{y}(k)\right)^{T} P_{2}\left(\eta_{2}(k)+e_{y}(k)\right) \\
& -e_{y}^{T}(k) P_{2} e_{y}(k)=\xi^{T}(k) \Xi_{1} \xi(k), \\
& \Delta V_{2}(k)=e_{x}^{T}(k) Q_{1} e_{x}(k) \\
& -e_{x}^{T}\left(k-\delta_{m}\right) Q_{1} e_{x}\left(k-\delta_{m}\right) \\
& +e_{x}^{T}(k) Q_{3} e_{x}(k) \\
& -e_{x}^{T}\left(k-\delta_{M}\right) Q_{3} e_{x}\left(k-\delta_{M}\right) \\
& +\delta_{1} e_{x}^{T}(k) Q_{2} e_{x}(k) \\
& +\sum_{i=k+1-\delta(k+1)}^{k} e_{x}^{T}(i) Q_{2} e_{x}(i) \\
& -\sum_{i=k-\delta(k)}^{k-1} e_{x}^{T}(i) Q_{2} e_{x}(i) \\
& -\sum_{j=-\delta_{M}+1}^{-\delta_{m}} e_{x}^{T}(k+j) Q_{2} e_{x}(k+j) \\
& \leq e_{x}^{T}(k) Q_{1} e_{x}(k)+\left(1+\delta_{1}\right) e_{x}^{T}(k) Q_{2} e_{x}(k) \\
& +e_{x}^{T}(k) Q_{3} e_{x}(k) \\
& -e_{x}^{T}\left(k-\delta_{m}\right) Q_{1} e_{x}\left(k-\delta_{m}\right)
\end{aligned}
$$




$$
\begin{aligned}
& -e_{x}^{T}(k-\delta(k)) Q_{2} e_{x}(k-\delta(k)) \\
& -e_{x}^{T}\left(k-\delta_{M}\right) Q_{3} e_{x}\left(k-\delta_{M}\right) \\
= & \xi^{T}(k) \Xi_{2} \xi(k) .
\end{aligned}
$$

In the same way, we obtain

$$
\begin{aligned}
& \Delta V_{3}(k) \leq e_{y}^{T}(k) Q_{4} e_{y}(k)+\left(1+\tau_{1}\right) e_{y}^{T}(k) Q_{5} e_{y}(k) \\
& +e_{y}^{T}(k) Q_{6} e_{y}(k) \\
& -e_{y}^{T}\left(k-\tau_{m}\right) Q_{4} e_{y}\left(k-\tau_{m}\right) \\
& -e_{y}^{T}(k-\tau(k)) Q_{5} e_{y}(k-\tau(k)) \\
& -e_{y}^{T}\left(k-\tau_{M}\right) Q_{6} e_{y}\left(k-\tau_{M}\right) \\
& =\xi^{T}(k) \Xi_{3} \xi(k), \\
& \Delta V_{4}(k)=\tau_{1} g^{T}\left(e_{y}(k)\right) \operatorname{Rg}\left(e_{y}(k)\right) \\
& +\sum_{i=k+1-\tau(k+1)}^{k} g^{T}\left(e_{y}(i)\right) R g\left(e_{y}(i)\right) \\
& -\sum_{i=k-\tau(k)}^{k-1} g^{T}\left(e_{y}(i)\right) R g\left(e_{y}(i)\right) \\
& -\sum_{j=-\tau_{M}+1}^{-\tau_{M}} g^{T}\left(e_{y}(k+j)\right) R g\left(e_{y}(k+j)\right) \\
& \leq\left(1+\tau_{1}\right) g^{T}\left(e_{y}(k)\right) \operatorname{Rg}\left(e_{y}(k)\right) \\
& -g^{T}\left(e_{y}(k-\tau(k))\right) \operatorname{Rg}\left(e_{y}(k-\tau(k))\right) \\
& =\xi^{T}(k) \Xi_{4} \xi(k), \\
& \Delta V_{5}(k)=\delta_{1} \eta_{1}^{T}(k) Z_{1} \eta_{1}(k)-\sum_{i=k-\delta_{M}}^{k-\delta_{m}-1} \eta_{1}^{T}(i) Z_{1} \eta_{1}(i) \\
& +\delta_{m} \eta_{1}^{T}(k) Z_{2} \eta_{1}(k)-\sum_{i=k-\delta_{m}}^{k-1} \eta_{1}^{T}(i) Z_{2} \eta_{1}(i) \\
& +\tau_{1} \eta_{2}^{T}(k) Z_{3} \eta_{2}(k)-\sum_{i=k-\tau_{M}}^{k-\tau_{m}-1} \eta_{2}^{T}(i) Z_{3} \eta_{2}(i) \\
& +\tau_{m} \eta_{2}^{T}(k) Z_{4} \eta_{2}(k) \\
& -\sum_{i=k-\tau_{m}}^{k-1} \eta_{2}^{T}(i) Z_{4} \eta_{2}(i)
\end{aligned}
$$

$$
\begin{gathered}
\Delta V_{5}(k) \leq \eta_{1}^{T}(k)\left(\delta_{1} Z_{1}+\delta_{m} Z_{2}\right) \eta_{1}(k)+\eta_{2}^{T}(k)\left(\tau_{1} Z_{3}\right. \\
\left.+\tau_{m} Z_{4}\right) \eta_{2}(k)-\xi^{T}(k)\left[\frac{1}{\delta_{m}} \rho_{1} \widetilde{Z}_{2} \rho_{1}^{T}+\frac{1}{\delta_{1}} \rho_{7} \Theta_{1} \rho_{7}^{T}\right. \\
\left.+\frac{1}{\tau_{m}} \rho_{4} \widetilde{Z}_{4} \rho_{4}^{T}+\frac{1}{\tau_{1}} \rho_{8} \Theta_{2} \rho_{8}^{T}\right] \xi(k)=\xi^{T}(k) \Xi_{5} \xi(k) .
\end{gathered}
$$

$$
\begin{aligned}
& -\sum_{i=k-\delta_{M}}^{k-\delta_{m}-1} \eta_{1}^{T}(i) Z_{1} \eta_{1}(i)=-\sum_{i=k-\delta_{M}}^{k-\delta(k)-1} \eta_{1}^{T}(i) Z_{1} \eta_{1}(i) \\
& -\sum_{i=k-\delta(k)}^{k-\delta_{m}-1} \eta_{1}^{T}(i) Z_{1} \eta_{1}(i) \leq-\frac{1}{\delta_{M}-\delta_{m}} \xi^{T}(k) \\
& \cdot \rho_{2} \widetilde{Z}_{1} \rho_{2}{ }^{T} \xi(k)-\frac{\delta(k)-\delta_{m}}{\delta_{M}-\delta_{m}} \frac{1}{\delta_{M}-\delta(k)} \xi^{T}(k) \\
& \cdot \rho_{2} \widetilde{Z}_{1} \rho_{2}{ }^{T} \xi(k)-\frac{1}{\delta_{M}-\delta_{m}} \xi^{T}(k) \rho_{3} \widetilde{Z}_{1} \rho_{3}{ }^{T} \xi(k) \\
& -\frac{\delta_{M}-\delta(k)}{\delta_{M}-\delta_{m}} \frac{1}{\delta(k)-\delta_{m}} \xi^{T}(k) \rho_{3} \widetilde{Z}_{1} \rho_{3}{ }^{T} \xi(k) \\
& =-\frac{1}{\delta_{M}-\delta_{m}}\left[\frac{\delta_{M}-\delta_{m}}{\delta_{M}-\delta(k)} \xi^{T}(k) \rho_{2} \widetilde{Z}_{1} \rho_{2}{ }^{T} \xi(k)\right. \\
& \left.+\frac{\delta_{M}-\delta_{m}}{\delta(k)-\delta_{m}} \xi^{T}(k) \rho_{3} \widetilde{Z}_{1} \rho_{3}{ }^{T} \xi(k)\right] \leq-\frac{1}{\delta_{M}-\delta_{m}} \\
& \cdot \xi^{T}(k) \rho_{7} \Theta_{1} \rho_{7}{ }^{T} \xi(k) .
\end{aligned}
$$

Based on (35)-(37), we have 
From Assumption 1, for diagonal matrices $\Lambda_{1}, \Lambda_{2}$, and $\Lambda_{3}>0$ and a known matrix $L=\operatorname{diag}\left\{l_{1}, l_{2}, \ldots, l_{n}\right\}>0$, it is not difficult to see that the inequalities

$$
\begin{aligned}
& 2 g^{T}\left(e_{y}(k)\right) \Lambda_{1} g\left(e_{y}(k)\right)-2 e_{y}^{T}(k) L \Lambda_{1} g\left(e_{y}(k)\right) \\
& \quad \leq 0 \\
& 2 g^{T}\left(e_{y}(k-\tau(k))\right) \Lambda_{2} g\left(e_{y}(k-\tau(k))\right) \\
& \quad-2 e_{y}^{T}(k-\tau(k)) L \Lambda_{2} g\left(e_{y}(k-\tau(k))\right) \leq 0, \\
& 2\left[g\left(e_{y}(k)\right)-g\left(e_{y}(k-\tau(k))\right)\right]^{T} \\
& \cdot \Lambda_{3}\left[g\left(e_{y}(k)\right)-g\left(e_{y}(k-\tau(k))\right)\right] \\
& \quad-2\left[e_{y}(k)-e_{y}(k-\tau(k))\right]^{T} \\
& \cdot L \Lambda_{3} g\left(e_{y}(k)-e_{y}(k-\tau(k))\right) \leq 0
\end{aligned}
$$

hold. In addition, since $\eta_{1}(k)=e_{x}(k+1)-e_{x}(k), \eta_{2}(k)=$ $e_{y}(k+1)-e_{y}(k)$, by introducing relaxation matrices $N_{1}=$ $\operatorname{diag}\left\{N_{11}, N_{12}\right\}, N_{2}=\operatorname{diag}\left\{N_{21}, N_{12}\right\}, N_{3}=\operatorname{diag}\left\{N_{31}, N_{32}\right\}$, and $N_{4}=\operatorname{diag}\left\{N_{41}, N_{32}\right\}$ with appropriate dimensions, we obtain

$$
\begin{aligned}
& 2\left(\eta_{1}^{T}(k) N_{1}+e_{x}^{T}(k) N_{2}\right)\left[\left(\bar{A}_{1}-I_{2 n}\right) e_{x}(k)\right. \\
& \left.\quad+\bar{B}_{1} g\left(e_{y}(k-\tau(k))\right)+\bar{E}_{2} \varrho_{2}(k)-\eta_{1}(k)\right]=0, \\
& 2\left(\eta_{2}^{T}(k) N_{3}+e_{y}^{T}(k) N_{4}\right)\left[\left(\bar{C}_{1}-I_{2 n}\right) e_{y}(k)\right. \\
& \left.\quad+\bar{D}_{1} e_{x}(k-\delta(k))+\bar{E}_{2} \varrho_{4}(k)-\eta_{2}(k)\right]=0 .
\end{aligned}
$$

From $F^{T}(k) F(k) \leq I$, we have

$$
\varrho_{i}^{T}(k) \varrho_{i}(k) \leq \zeta_{i}^{T}(k) \zeta_{i}(k), \quad(i=2,4) .
$$

Then, there exist positive scalars $\varepsilon_{2}$ and $\varepsilon_{4}$ satisfying

$$
\varepsilon_{i}\left[\xi^{T}(k) \Psi_{i}^{T}(k) \Psi_{i}(k) \xi(k)-\varrho_{i}^{T}(k) \varrho_{i}(k)\right] \geq 0 .
$$

Now, combining (32)-(45) and taking mathematical expectation, one can obtain the following inequality:

$$
\begin{aligned}
\mathbb{E} & {[\Delta V(k)] } \\
& \leq \mathbb{E}\left[\xi^{T}(k)\left(\sum_{i=1}^{9} \Xi_{i}+\varepsilon_{2} \Psi_{2}^{T} \Psi_{2}+\varepsilon_{4} \Psi_{4}^{T} \Psi_{4}\right) \xi(k)\right] \\
& <0 .
\end{aligned}
$$

Using the Schur complement Lemma, it can be shown that the considered uncertain system (24) is globally asymptotically stable in mean square sense, which completes the proof of the theorem.

Remark 7. It is worthwhile to mention that two constraint conditions (i.e., (39)-(40)) on feedback regulatory function were used on dealing with the state estimation problem for discrete GRNs with time-varying delays. In this paper, we add inequality (41) with hope to reduce the conservativeness introduced by time delays.

Now, we are in a position to deal with the nonfragile estimator design for GRNs (4) with time-varying delays and randomly occurring uncertainties.

Theorem 8. For given positive scalars $\delta_{m}, \delta_{M}, \tau_{m}$, and $\tau_{M}$, system (8) is said to be a globally robustly asymptotic state estimator of GRNs (4), if there exist scalars $\varepsilon_{i}>0(i=$ $1,2,3,4)$, matrices $P_{1}>0, P_{2}>0, R>0, Z_{j}>0(j=$ $1,2,3,4)$, and $Q_{l}>0(l=1,2, \ldots, 6)$, positive diagonal

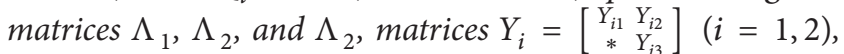
and block diagonal matrices $N_{1}, N_{2}, N_{3}, N_{4}, X_{1}$, and $X_{3}$ with appropriate dimensions, such that the following LMIs hold:

$$
\widetilde{\Sigma}=\left[\begin{array}{ccccc}
\sum_{i=1}^{11} \Xi_{i} & \varepsilon_{1} \Psi_{1}^{T} & \varepsilon_{2} \Psi_{2}^{T} & \varepsilon_{3} \Psi_{3}^{T} & \varepsilon_{4} \Psi_{4}^{T} \\
* & -\varepsilon_{1} I & 0 & 0 & 0 \\
* & * & -\varepsilon_{2} I & 0 & 0 \\
* & * & * & -\varepsilon_{3} I & 0 \\
* & * & * & * & -\varepsilon_{4} I
\end{array}\right]<0
$$

with

$$
\begin{aligned}
& \Psi_{1}=\left[\begin{array}{llll}
\alpha_{1} \bar{M}_{1} & 0_{n \times 10 n} & \beta_{2} \bar{M}_{2} & 0_{n \times 10 n}
\end{array}\right], \\
& \Psi_{2}=\left[\begin{array}{ll}
\gamma_{1} \bar{M}_{5} & 0 \\
n \times 21 n
\end{array}\right] \text {, } \\
& \Psi_{3}=\left[\begin{array}{lllll}
0_{n \times n} & \alpha_{2} \bar{M}_{4} & 0_{n \times 2 n} & \beta_{1} \bar{M}_{3} & 0_{n \times 17 n}
\end{array}\right], \\
& \Psi_{4}=\left[\begin{array}{lll}
0_{n \times 4 n} & \gamma_{2} \bar{M}_{6} & 0_{n \times 17 n}
\end{array}\right], \\
& \Xi_{10}=2\left[e_{9} N_{1}+e_{1} N_{2}\right] \bar{E}_{1} e_{21}^{T} \\
& +2\left[e_{10} N_{3}+e_{5} N_{4}\right] \bar{E}_{1} e_{22}^{T}, \\
& \Xi_{11}=-e_{21} \varepsilon_{1} I_{2 n} e_{21}^{T}-e_{22} \varepsilon_{3} I_{2 n} e_{22}^{T} \text {, }
\end{aligned}
$$

and the other parameters are defined as in Theorem 6. Moreover, the estimation gain matrices are determined by

$$
\begin{aligned}
& K_{1}=N_{12}^{-1} X_{1}, \\
& K_{2}=N_{32}^{-1} X_{3} .
\end{aligned}
$$


Proof. The proof of this theorem follows from Theorem 6 directly. According to (13), for any matrices $N_{1}, N_{2}, N_{3}$, and $N_{4}$, we have

$$
\begin{aligned}
& 2\left(\eta_{1}^{T}(k) N_{1}+e_{x}^{T}(k) N_{2}\right)\left[\left(\bar{A}_{1}-I_{2 n}\right) e_{x}(k)\right. \\
& \quad+\bar{B}_{1} g\left(e_{y}(k-\tau(k))\right)+\bar{E}_{1} \varrho_{1}(k)+\bar{E}_{2} \varrho_{2}(k) \\
& \left.\quad-\eta_{1}(k)\right]=0, \\
& 2\left(\eta_{2}^{T}(k) N_{3}+e_{y}^{T}(k) N_{4}\right)\left[\left(\bar{C}_{1}-I_{2 n}\right) e_{y}(k)\right. \\
& \left.\quad+\bar{D}_{1} e_{x}(k-\delta(k))+\bar{E}_{1} \varrho_{3}(k)+\bar{E}_{2} \varrho_{4}(k)-\eta_{2}(k)\right] \\
& \quad=0 .
\end{aligned}
$$

Similarly, we get

$$
\varrho_{i}^{T}(k) \varrho_{i}(k) \leq \zeta_{i}^{T}(k) \zeta_{i}(k), \quad(i=1,2,3,4)
$$

Then, for positive scalars $\varepsilon_{i}$, we have

$$
\begin{aligned}
& \varepsilon_{i}\left[\tilde{\xi}^{T}(k) \Psi_{i}^{T}(k) \Psi_{i}(k) \tilde{\xi}(k)-\varrho_{i}^{T}(k) \varrho_{i}(k)\right] \geq 0, \\
&(i=1,2,3,4),
\end{aligned}
$$

with

$$
\begin{aligned}
& \tilde{\xi}^{T}(k)=\left[e_{x}^{T}(k), e_{x}^{T}(k-\delta(k)), e_{x}^{T}\left(k-\delta_{m}\right),\right. \\
& e_{x}^{T}\left(k-\delta_{M}\right), e_{y}^{T}(k), e_{y}^{T}(k-\tau(k)), e_{y}^{T}\left(k-\tau_{m}\right), \\
& e_{y}^{T}\left(k-\tau_{M}\right), \eta_{1}^{T}(k), \eta_{2}^{T}(k), g^{T}\left(e_{y}(k)\right), \\
& g^{T}\left(e_{y}(k-\tau(k))\right), \chi_{1}^{T}(k), \chi_{2}^{T}(k), \chi_{3}^{T}(k), \chi_{4}^{T}(k), \\
& \left.\chi_{5}^{T}(k), \chi_{6}^{T}(k), \varrho_{2}^{T}(k), \varrho_{4}^{T}(k), \varrho_{1}^{T}(k), \varrho_{3}^{T}(k)\right] .
\end{aligned}
$$

Combining the above and taking the mathematical expectation, one has

$$
\mathbb{E}[\Delta V(k)] \leq \mathbb{E}\left[\widetilde{\xi}^{T}(k) \widetilde{\Sigma} \widetilde{\xi}(k)\right]
$$

By using the Schur complement Lemma, it is easy to conclude that the proof of this theorem is complete.

Remark 9. In this paper, a nonfragile state estimation algorithm has been presented for discrete-time GRNs with time-varying delays and randomly occurring uncertainties. It is worth mentioning that it is the first time to use the discrete Wirtinger-based inequality and the reciprocally convex inequality to handle the state estimation problem for discrete-time delayed GRNs subject to randomly occurring uncertainties and estimator gain perturbations. In particular, the reciprocally convex approach, the discrete Wirtingerbased inequality, and the free-weighting matrix method are fully utilized in order to reduce the conservativeness of the developed estimation criterion. Besides, by introducing two new zero equations (42) and (43), the free-weighting matrices $N_{1}, N_{2}, N_{3}$, and $N_{4}$ have been introduced in the main results with hope to further reduce the possible conservativeness induced by time-varying delays, which could be utilized to deal with related state estimation problems for complex systems with time delays.

Remark 10. It should be noted that the presented method in this paper is based on LMIs. The standard LMI system has a polynomial-time complexity bounded by $O(M N)$, where $M$ is the total row size of the LMI system and $N$ is the total number of scalar decision variables. For example, let us look at the LMIs condition for the addressed GRNs (as described in Theorem 6); we have $M=44 n$ and $N=2+13 n(n+1) / 2+$ $2\left(n(n+1)+n^{2}\right)+3 n+8 n^{2}+2 n^{2}=(1 / 2)\left(41 n^{2}+23 n+4\right)$. Therefore, the computational complexity of the LMI-based state estimation criterion can be represented by $O\left(902 n^{3}+\right.$ $\left.506 n^{2}+88 n\right)$.

Remark 11. It is worthwhile to point out that our major focus is on the nonfragile state estimation problem for discretetime GRNs subject to time-varying delays and randomly occurring uncertainties. Due to such a complicated system, we have decided to carry out research on the feasibility problem with satisfactory performance requirements for addressed GRNs; that is, we made great efforts to propose a new state estimation method against the above-mentioned phenomena. Nevertheless, in case that the convergence precision of the proposed state estimation method becomes a concern, some additional conditions can be provided, which constitutes one of our future research topics.

\section{Numerical Simulations}

In this section, we present two numerical examples to illustrate the effectiveness and correctness of the proposed state estimation scheme.

Example 1. Consider three-node GRNs with the following parameters:

$$
\begin{aligned}
A & =D=0.3 I, \\
C & =0.1 I, \\
F(k) & =\operatorname{diag}\{\sin (k), \cos (k), 0\}, \\
B & =0.5 \times\left[\begin{array}{ccc}
0 & 0 & -1 \\
-1 & 0 & 1 \\
0 & -1 & 0
\end{array}\right],
\end{aligned}
$$




$$
\begin{aligned}
& W_{1}=\left[\begin{array}{ccc}
0.3 & 0.5 & 0 \\
0.2 & 0.1 & 0.15
\end{array}\right], \\
& W_{2}=\left[\begin{array}{ccc}
0.4 & 0.1 & -0.5 \\
-0.2 & 0.1 & 0.3
\end{array}\right], \\
& E_{2}=\left[\begin{array}{ccc}
0.2 & 0.3 & 0 \\
0.1 & 0 & 0.2 \\
0 & 0.3 & 0
\end{array}\right], \\
& M_{5}=\left[\begin{array}{cc}
-0.3 & -0.05 \\
-0.4 & 0.06 \\
-0.1 & 0.02
\end{array}\right], \\
& M_{6}=\left[\begin{array}{cc}
0.1 & 0.03 \\
0.02 & 0.1 \\
0.03 & 0.1
\end{array}\right] .
\end{aligned}
$$

The nonlinear genetic regulatory function $g(s)$ is taken to be as usual Hill function. That is, $g(s)=s^{2} /\left(1+s^{2}\right)$. Then, it is easy to see that

$$
L=\operatorname{diag}\{0.65,0.65,0.65\} .
$$

This means that Assumption 1 holds. Moreover, assume $\delta_{m}=$ $1, \delta_{M}=5, \tau_{m}=2, \tau_{M}=4, \gamma_{1}=0.3$, and $\gamma_{2}=0.7$. By using the LMIs control Toolbox in Matlab to solve the LMIs in Theorem 6, we can obtain feasible solutions. Due to space consideration, only the following few parameters are listed: $\varepsilon_{2}=1.1713 ; \varepsilon_{4}=1.0898$; the nonfragile state estimator can be designed with the estimator gain matrices described by

$$
\begin{aligned}
& K_{1}=\left[\begin{array}{cc}
0.0968 & -0.1305 \\
-0.1321 & 0.2728 \\
0.0909 & -0.2096
\end{array}\right], \\
& K_{2}=\left[\begin{array}{cc}
-0.2167 & 0.1296 \\
0.5065 & 0.8443 \\
-0.0759 & 0.1388
\end{array}\right] .
\end{aligned}
$$

The numerical simulation affirms our theoretical results. Figure 1 plots the trajectories of mRNA concentrations, its estimates, and the estimation errors with initial conditions $\phi(k)=\left[\begin{array}{lll}0.2 & 0.2 & 0.4\end{array}\right]^{T}$ and $\widehat{\phi}(k)=\left[\begin{array}{lll}0.8 & 0.5 & 0.9\end{array}\right]^{T}$. Figure 2 depicts the trajectories of protein concentrations, its estimates, and the estimation errors with initial conditions $\psi(k)=\left[\begin{array}{lll}0.1 & 0.5 & 0.3\end{array}\right]^{T}$ and $\widehat{\psi}(k)=\left[\begin{array}{lll}0.5 & 0.8 & 0.3\end{array}\right]^{T}$. It follows from Figures 1 and 2 that the estimation error between the original GRNs and its estimation approaches zero asymptotically. In addition, in order to illustrate the advantage of the main results, we can set $E_{2}=0$. Then, error system (24) is equivalent to system (6) in [27]. Subsequently, it is not difficult to check that when the feedback regulation delay $\tau(k)$ and the translation delay $\delta(k)$ satisfy $2 \leq \tau(k) \leq 5$ and $2 \leq \delta(k) \leq 9$, respectively, LMI (25) in Theorem 6 of our paper is feasible while LMI condition (7) in [27] is infeasible. Therefore, it is concluded that our results are less conservative than those in [27]. The major reason is that we have made additional efforts to propose new results with more information of time delays by taking the advantage of the discrete-time Wirtinger-based inequality, the reciprocally convex combination approach, and the free-weight matrix technique. On the other hand, it should be mentioned that, compared to [27] without consideration of estimator gain perturbations, the developed state estimation approach is more applicable.

Example 2. To demonstrate the effectiveness of Theorem 8, we consider the following parameters as in Example 1, with

$$
\begin{aligned}
& E_{1}=\left[\begin{array}{ccc}
0.2 & 0 & 0.1 \\
0.2 & -0.1 & 0 \\
0 & 0.2 & 0.3
\end{array}\right], \\
& E_{2}=\left[\begin{array}{ccc}
0.1 & 0 & -0.2 \\
0.2 & 0.1 & 0 \\
0.1 & 0 & 0.3
\end{array}\right], \\
& M_{1}=\left[\begin{array}{ccc}
0.2 & 0.05 & -0.3 \\
0.2 & -0.1 & 0 \\
0.2 & 0.3 & 0.6
\end{array}\right] \text {, } \\
& M_{2}=\left[\begin{array}{ccc}
0.1 & 0.3 & 0 \\
0.2 & 0.1 & 0.2 \\
0.1 & 0 & 0.3
\end{array}\right], \\
& M_{3}=\left[\begin{array}{ccc}
-0.1 & 0 & -0.06 \\
0 & 0.1 & 0.2 \\
0.3 & 0.2 & -0.2
\end{array}\right] \text {, } \\
& M_{4}=\left[\begin{array}{ccc}
0.2 & 0.1 & 0.2 \\
-0.5 & 0.2 & 0.1 \\
-0.3 & 0.2 & 0
\end{array}\right] \text {, } \\
& M_{5}=\left[\begin{array}{cc}
-0.1 & 0.02 \\
0.05 & 0.1 \\
0.1 & 0.02
\end{array}\right] \text {, } \\
& M_{6}=\left[\begin{array}{cc}
0.2 & 0.03 \\
0.3 & 0.1 \\
-0.1 & 0
\end{array}\right] \text {. }
\end{aligned}
$$



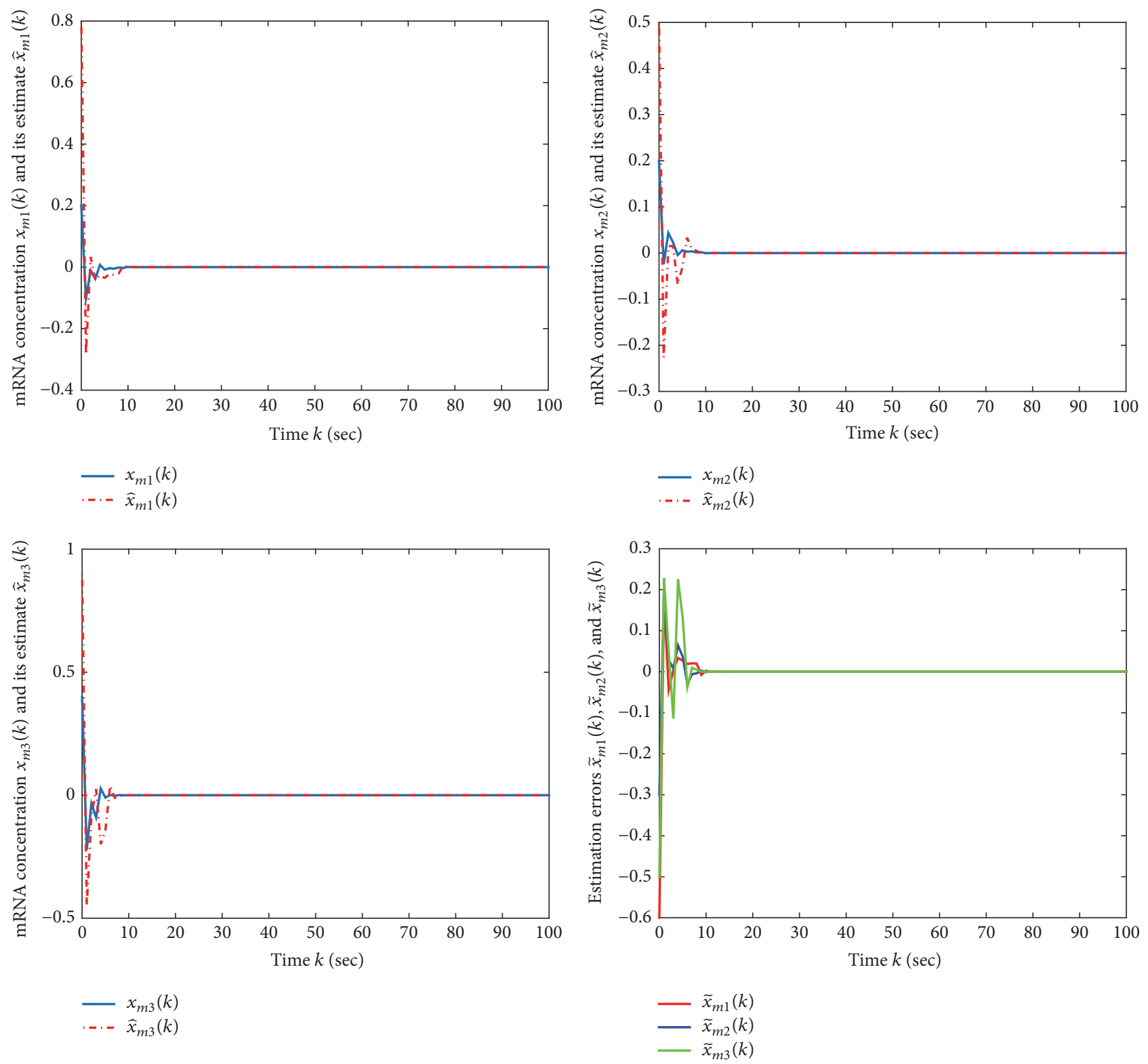

FIgURE 1: mRNA concentrations, its estimates, and the estimation error of Example 1.

Let the delays $\delta(k)$ and $\tau(k)$ vary randomly within the intervals $[2,5]$. The mathematical expectations of Bernoullidistributed white sequences can be taken as $\alpha_{1}=0.5, \alpha_{2}=$ $0.4, \beta_{1}=0.3, \beta_{2}=0.2, \gamma_{1}=0.4$, and $\gamma_{2}=0.3$. By solving the LMIs in Theorem 8 with the help of Matlab LMI toolbox, we can obtain feasible solutions and scalars $\varepsilon_{1}=0.1385$, $\varepsilon_{2}=0.6089, \varepsilon_{3}=0.0910$, and $\varepsilon_{4}=0.5866$. According to Theorem 8 , the nonfragile state estimator with randomly occurring uncertainties can be designed with the estimator gain matrices defined as follows:

$$
K_{1}=\left[\begin{array}{cc}
0.1198 & -0.2160 \\
-0.2708 & 0.4029 \\
0.1412 & -0.3462
\end{array}\right] \text {, }
$$

$$
K_{2}=\left[\begin{array}{ll}
0.0842 & 0.0778 \\
0.3287 & 0.5749 \\
0.0143 & 0.1117
\end{array}\right]
$$

The simulation results are shown in Figures 3 and 4 . The actual state responses $x(k)$ and $y(k)$, their estimates $\widehat{x}(k)$ and $\widehat{y}(k)$, and the estimation error are depicted in Figures 3 and 4. The initial conditions are taken as $\phi(k)=\left[\begin{array}{lll}0.5 & 0.3 & 0.6\end{array}\right]^{T}$, $\widehat{\phi}(k)=\left[\begin{array}{lll}0.3 & 0.8 & 0.2\end{array}\right]^{T}, \psi(k)=\left[\begin{array}{lll}0.7 & 0.3 & 0.4\end{array}\right]^{T}$, and $\widehat{\psi}(k)=\left[\begin{array}{lll}0.2 & 0.9 & 0.8\end{array}\right]^{T}$. It is also observed from Figures 3 and 4 that the estimation error between the original GRNs 

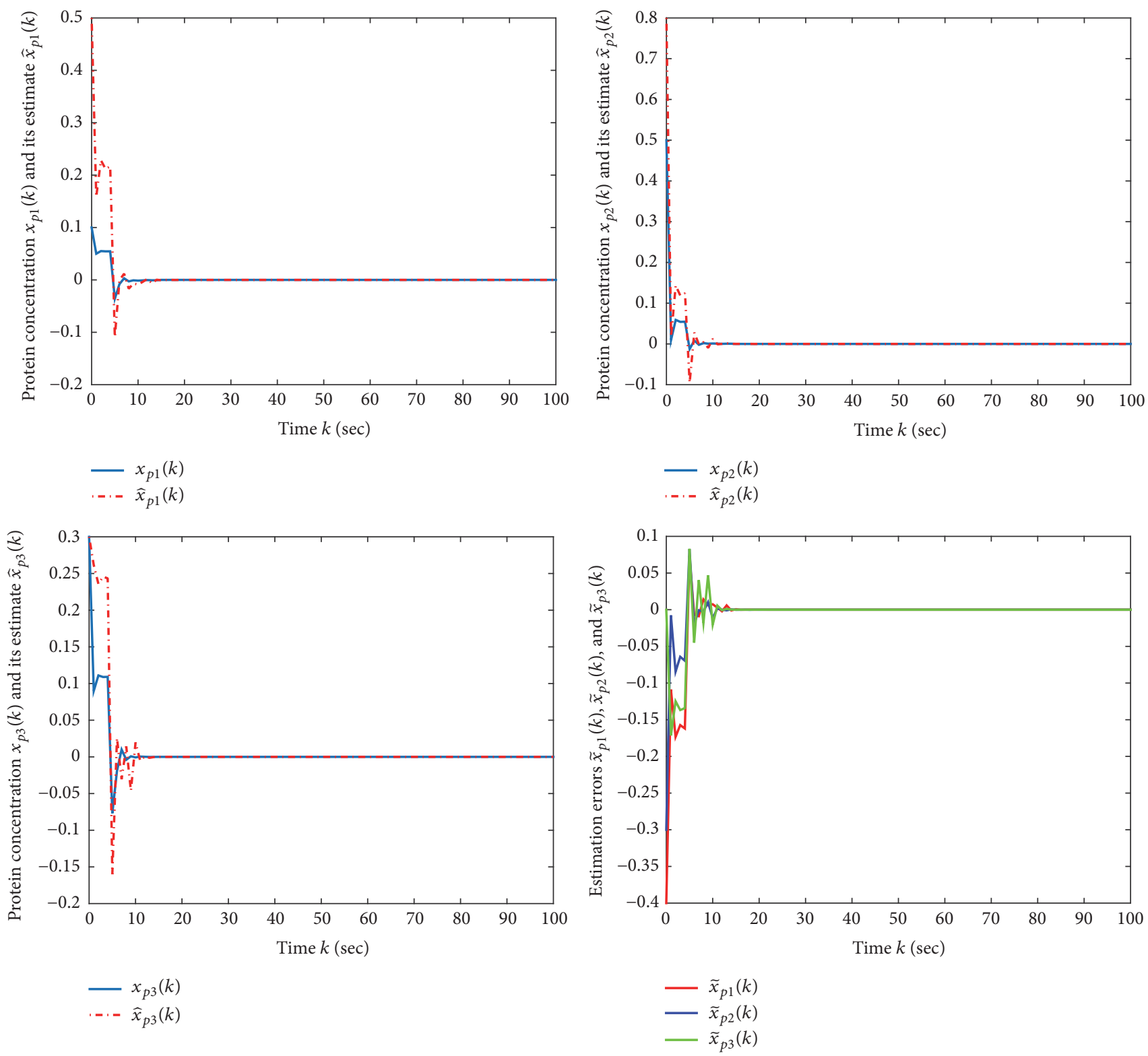

Figure 2: Protein concentrations, its estimates, and the estimation error of Example 1.

and the estimator system approaches zero asymptotically. It should be noticed that when we do not consider the nonfragility of estimator gain perturbations, the admissibility of upper bounds of time delays (under different lower bounds of time delays) guaranteeing the globally asymptotical stability of the resulting estimation error system for the addressed GRNs can be obtained and listed in Table 1 by using Theorem 2 in [27] and Theorem 8 in this paper, respectively, which further illustrate the advantages and less conservatism of the newly proposed result.

\section{Conclusion}

In this paper, we have investigated the nonfragile state estimator design for a class of the discrete-time GRNs
TABLE 1: Admissible upper bound $\delta_{M}$ with different $\delta_{m}$, when $\tau_{m}=2$ and $\tau_{M}=5$.

\begin{tabular}{lccccc}
\hline$\delta_{m}$ & 2 & 4 & 6 & 8 & 10 \\
\hline [[27], Theorem 2] & 6 & 8 & 10 & 12 & 14 \\
Theorem 8 & 7 & 9 & 11 & 13 & 15 \\
\hline
\end{tabular}

with interval time-varying delays and randomly occurring uncertainties. The norm-bounded uncertainties enter into the GRNs in random ways, and such randomly occurring uncertainties have been characterized by certain mutually uncorrelated Bernoulli-distributed white noise sequences. Under these circumstances, the state estimator has been 

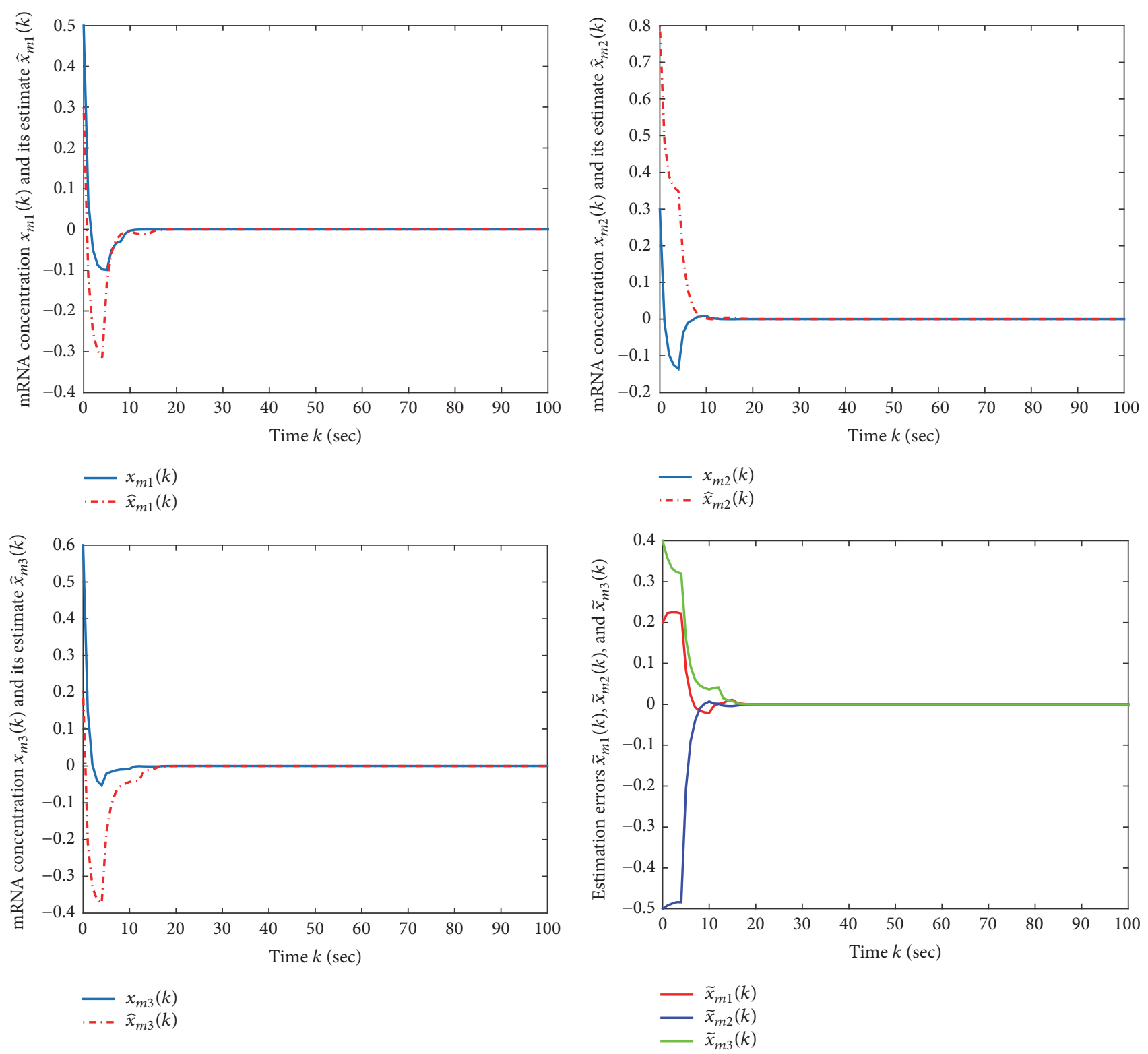

FIGURE 3: mRNA concentrations, its estimates, and the estimation error of Example 2.

designed to estimate the true concentration of the mRNA and the protein from available measurements. By constructing a Lyapunov-Krasovskii functional, new delay-dependent estimation criterion has been obtained in terms of LMIs by using the discrete-time Wirtinger-based inequality and the reciprocally convex approach. A new constraint condition on feedback regulatory function has been used with hope to reduce the conservativeness of the estimation criterion. Then, the desired state estimation algorithm has been provided in view of the solutions to LMIs, which can ensure that the estimation error dynamics are globally asymptotically stable. Finally, numerical simulations have been given to illustrate the applicability and usefulness of the developed theoretical results. It is worth mentioning that the proposed method in this paper can be extended to address the nonfragile state estimation problems for Markovian jump systems as in $[40,41]$ with different types of time delays and time-varying systems in [42-44] with unreliable measurements, which constitute interesting topic for future research.

\section{Conflicts of Interest}

The authors declare that there are no conflicts of interest regarding the publication of this paper. 

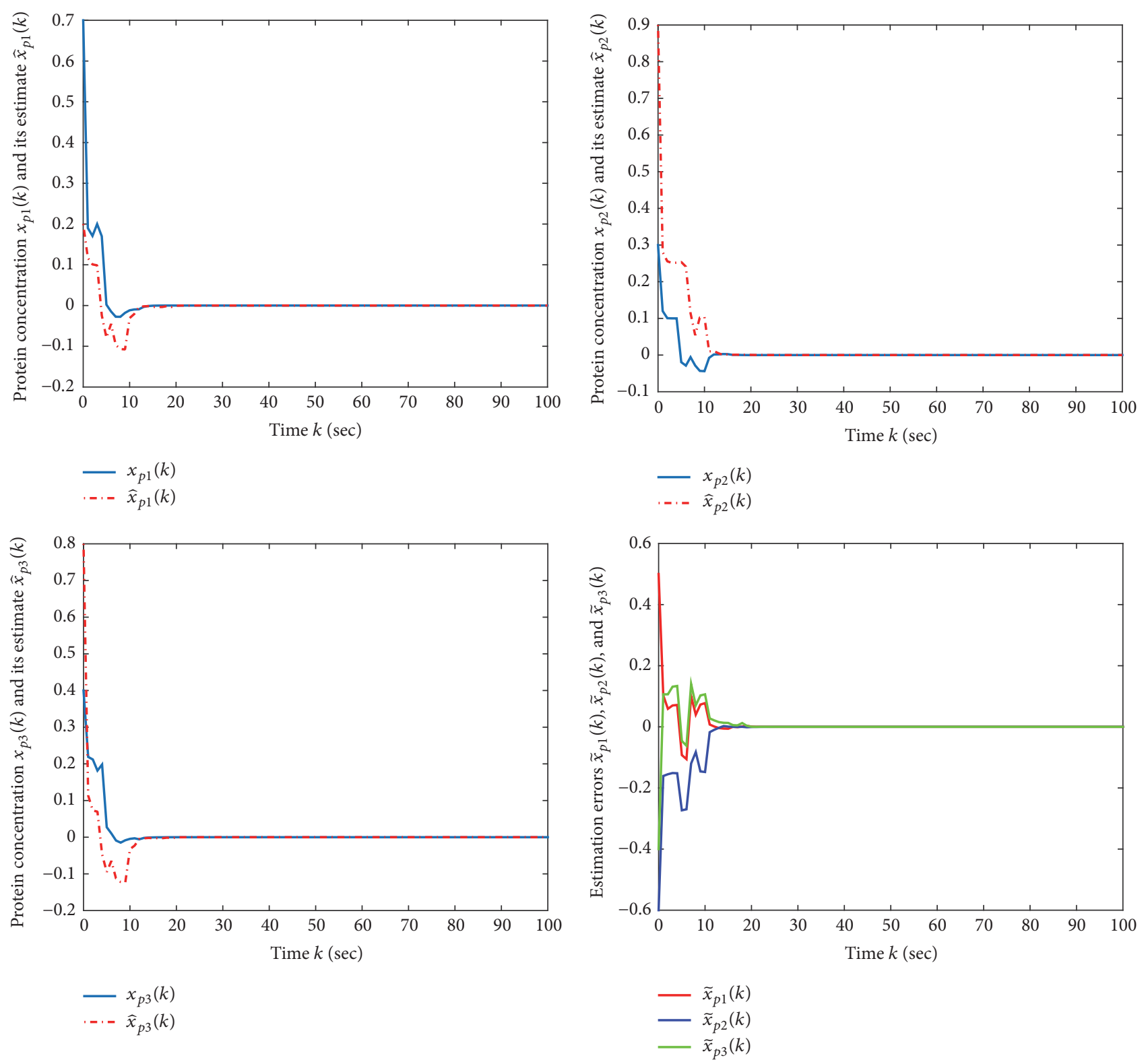

Figure 4: Protein concentrations, its estimates, and the estimation error of Example 2.

\section{Acknowledgments}

This work was supported in part by the National Natural Science Foundation of China under Grants 11401141 and 11271103, Heilongjiang Provincial Natural Science Foundation for Youths under Grant QC2013C001, Heilongjiang Provincial Postdoctoral Science Foundation under Grant LBH-Z15098, and Natural Science Foundation of Heilongjiang Educational Committee under Grant 12531099.

\section{References}

[1] L. Chen and K. Aihara, "Stability of genetic regulatory networks with time delay," IEEE Transactions on Circuits and Systems. I. Fundamental Theory and Applications, vol. 49, no. 5, pp. 602$608,2002$.
[2] G. Chesi and Y. S. Hung, "Stability analysis of uncertain genetic sum regulatory networks," Automatica. A Journal of IFAC, the International Federation of Automatic Control, vol. 44, no. 9, pp. 2298-2305, 2008.

[3] F. Ren and J. Cao, "Asymptotic and robust stability of genetic regulatory networks with time-varying delays," Neurocomputing, vol. 71, no. 4-6, pp. 834-842, 2008.

[4] M. Chaves, E. D. Sontag, and R. Albert, "Methods of robustness analysis for Boolean models of gene control networks," IEE Proceedings: Systems Biology, vol. 153, no. 4, pp. 154-167, 2006.

[5] M. Han, Y. Liu, and Y. Tu, "Controllability of Boolean control networks with time delays both in states and inputs," Neurocomputing, vol. 129, pp. 467-475, 2014.

[6] H. de Jong, "Modeling and simulation of genetic regulatory systems: a literature review," Journal of Computational Biology, vol. 9, no. 1, pp. 67-103, 2002. 
[7] I. Ivanov and E. R. Dougherty, "Modelling genetic regulatory networks: continuous or discrete," Journal of Biological Systems, vol. 2, no. 2, pp. 219-229, 2011.

[8] Y. Liu, F. E. Alsaadi, X. Yin, and Y. Wang, "Robust Ho filtering for discrete nonlinear delayed stochastic systems with missing measurements and randomly occurring nonlinearities," International Journal of General Systems, vol. 44, no. 2, pp. 169-181, 2015.

[9] X. Zhang, L. Wu, and S. Cui, "An improved integral inequality to stability analysis of genetic regulatory networks with interval time-varying delays," IEEE/ACM Transactions on Computational Biology and Bioinformatics, vol. 12, no. 2, pp. 398-409, 2015.

[10] W. Zhang, J.-A. Fang, and Y. Tang, "New robust stability analysis for genetic regulatory networks with random discrete delays and distributed delays," Neurocomputing, vol. 74, no. 14-15, pp. 2344-2360, 2011.

[11] R. Rakkiyappan, S. Lakshmanan, and P. Balasubramaniam, "Delay-probability-distribution-dependent stability of uncertain stochastic genetic regulatory networks with time-varying delays," Circuits, Systems, and Signal Processing, vol. 32, no. 3, pp. 1147-1177, 2013.

[12] J. Hu, Z. Wang, B. Shen, and H. Gao, "Gain-constrained recursive filtering with stochastic nonlinearities and probabilistic sensor delays," IEEE Transactions on Signal Processing, vol. 61, no. 5, pp. 1230-1238, 2013.

[13] Y. Wang, J. Cao, and L. Li, "Global robust power-rate stability of delayed genetic regulatory networks with noise perturbations," Cognitive Neurodynamics, vol. 4, no. 1, pp. 81-90, 2010.

[14] J. Cao and F. Ren, "Exponential stability of discrete-time genetic regulatory networks with delays," IEEE Transactions on Neural Networks, vol. 19, no. 3, pp. 520-523, 2008.

[15] Q. Li, B. Shen, Y. Liu, and F. E. Alsaadi, "Event-triggered $\mathrm{H}_{\infty}$ state estimation for discrete-time stochastic genetic regulatory networks with Markovian jumping parameters and timevarying delays," Neurocomputing, vol. 174, pp. 912-920, 2016.

[16] K. Mathiyalagan and R. Sakthivel, "Robust stabilization and $\mathrm{H}_{\infty}$ control for discrete-time stochastic genetic regulatory networks with time delays," Canadian Journal of Physics, vol. 90, no. 10, pp. 939-953, 2012.

[17] K. Mathiyalagan, R. Sakthivel, and S. M. Anthoni, "New robust passivity criteria for discrete-time genetic regulatory networks with Markovian jumping parameters," Canadian Journal of Physics, vol. 90, no. 2, pp. 107-118, 2012.

[18] X. Wan, L. Xu, H. Fang, and F. Yang, "Robust stability analysis for discrete-time genetic regulatory networks with probabilistic time delays," Neurocomputing, vol. 124, no. 2, pp. 72-80, 2014.

[19] Y. Zhao, J. Shen, and D. Chen, "New stability criterion for discrete-time genetic regulatory networks with time-varying delays and stochastic disturbances," Mathematical Problems in Engineering, Article ID 7634680, Art. ID 7634680, 13 pages, 2016.

[20] J. Hu, Z. Wang, D. Chen, and F. E. Alsaadi, "Estimation, filtering and fusion for networked systems with network-induced phenomena: new progress and prospects," Information Fusion, vol. 31, pp. 65-75, 2016.

[21] S. Lakshmanan, J. H. Park, H. Y. Jung, P. Balasubramaniam, and S. M. Lee, "Design of state estimator for genetic regulatory networks with time-varying delays and randomly occurring uncertainties," BioSystems, vol. 111, no. 1, pp. 51-70, 2013.
[22] P. Balasubramaniam and L. J. Banu, "Robust state estimation for discrete-time genetic regulatory network with random delays," Neurocomputing, vol. 122, pp. 349-369, 2013.

[23] J. Hu, Z. Wang, B. Shen, and H. Gao, "Quantised recursive filtering for a class of nonlinear systems with multiplicative noises and missing measurements," International Journal of Control, vol. 86, no. 4, pp. 650-663, 2013.

[24] J. Hu, Z. Wang, H. Gao, and L. K. Stergioulas, "Probabilityguaranteed $\mathrm{H} \infty$ finite-horizon filtering for a class of nonlinear time-varying systems with sensor saturations," Systems \& Control Letters, vol. 61, no. 4, pp. 477-484, 2012.

[25] R. Anbuvithya, K. Mathiyalagan, R. Sakthivel, and P. Prakash, "Sampled-data state estimation for genetic regulatory networks with time-varying delays," Neurocomputing, vol. 151, no. 2, pp. 737-744, 2015.

[26] J. Hu, Z. Wang, S. Liu, and H. Gao, "A variance-constrained approach to recursive state estimation for time-varying complex networks with missing measurements," Automatica. A Journal of IFAC, the International Federation of Automatic Control, vol. 64, pp. 155-162, 2016.

[27] R. Sakthivel, K. Mathiyalagan, S. Lakshmanan, and J. H. Park, "Robust state estimation for discrete-time genetic regulatory networks with randomly occurring uncertainties," Nonlinear Dynamics. An International Journal of Nonlinear Dynamics and Chaos in Engineering Systems, vol. 74, no. 4, pp. 1297-1315, 2013.

[28] J. Hu, D. Chen, and J. Du, "State estimation for a class of discrete nonlinear systems with randomly occurring uncertainties and distributed sensor delays," International Journal of General Systems, vol. 43, no. 3-4, pp. 387-401, 2014.

[29] H. Dong, Z. Wang, S. X. Ding, and H. Gao, "Finite-horizon reliable control with randomly occurring uncertainties and nonlinearities subject to output quantization," Automatica. A Journal of IFAC, the International Federation of Automatic Control, vol. 52, pp. 355-362, 2015.

[30] J. Hu, Z. Wang, H. Gao, and L. K. Stergioulas, "Robust sliding mode control for discrete stochastic systems with mixed time delays, randomly occurring uncertainties, and randomly occurring nonlinearities," IEEE Transactions on Industrial Electronics, vol. 59, no. 7, pp. 3008-3015, 2012.

[31] T. H. Lee, J. H. Park, Z.-G. Wu, S.-C. Lee, and D. H. Lee, "Robust $r H \infty$ decentralized dynamic control for synchronization of a complex dynamical network with randomly occurring uncertainties," Nonlinear Dynamics. An International Journal of Nonlinear Dynamics and Chaos in Engineering Systems, vol. 70, no. 1, pp. 559-570, 2012.

[32] J. Hu, S. Liu, D. Ji, and S. Li, "On co-design of filter and fault estimator against randomly occurring nonlinearities and randomly occurring deception attacks," International Journal of General Systems, vol. 45, no. 5, pp. 619-632, 2016.

[33] L. H. Keel and S. P. Bhattacharyya, "Robust, fragile, or optimal?" Institute of Electrical and Electronics Engineers. Transactions on Automatic Control, vol. 42, no. 8, pp. 1098-1105, 1997.

[34] Y. Yajing, D. Hongli, Z. Wangb, W. Rena, and F. E. Alsaadic, "Design of non-fragile state estimators for discrete time-delayed neural networks with parameter uncertainties," Neurocomputing, vol. 182, pp. 18-24, 2016.

[35] J. Hu, J. Liang, D. Chen, D. Ji, and J. Du, "A recursive approach to non-fragile filtering for networked systems with stochastic uncertainties and incomplete measurements," Journal of the Franklin Institute. Engineering and Applied Mathematics, vol. 352, no. 5, pp. 1946-1962, 2015. 
[36] N. Hou, H. Dong, Z. Wang, W. Ren, and F. E. Alsaadi, "Nonfragile state estimation for discrete Markovian jumping neural networks," Neurocomputing, vol. 179, pp. 238-245, 2016.

[37] P. T. Nam, P. N. Pathirana, and H. Trinh, "Discrete Wirtingerbased inequality and its application," Journal of the Franklin Institute. Engineering and Applied Mathematics, vol. 352, no. 5, pp. 1893-1905, 2015.

[38] S. Boyd, L. El Ghaoui, E. Feron, and V. Balakrishnan, Linear matrix inequalities in system and control theory, vol. 15 of SIAM Studies in Applied Mathematics, Society for Industrial and Applied Mathematics (SIAM), Philadelphia, Pa, USA, 1994.

[39] P. Park, J. W. Ko, and C. Jeong, "Reciprocally convex approach to stability of systems with time-varying delays," Automatica. A Journal of IFAC, the International Federation of Automatic Control, vol. 47, no. 1, pp. 235-238, 2011.

[40] L. Lu, B. He, C. Man, and S. Wang, "Robust state estimation for Markov jump genetic regulatory networks based on passivity theory," Complexity, vol. 21, no. 5, pp. 214-223, 2016.

[41] Y. Liu, W. Liu, M. A. Obaid, and I. A. Abbas, "Exponential stability of Markovian jumping Cohen-Grossberg neural networks with mixed mode-dependent time-delays," Neurocomputing, vol. 177, pp. 409-415, 2016.

[42] J. Zhang, L. Ma, and Y. Liu, "Passivity analysis for discretetime neural networks with mixed time-delays and randomly occurring quantization effects," Neurocomputing, vol. 216, pp. 657-665, 2016.

[43] J. Hu, Z. Wang, F. E. Alsaadi, and T. Hayat, "Event-based filtering for time-varying nonlinear systems subject to multiple missing measurements with uncertain missing probabilities," Information Fusion, vol. 38, pp. 74-83, 2017.

[44] S. Liu, G. Wei, Y. Song, and Y. Liu, "Extended Kalman filtering for stochastic nonlinear systems with randomly occurring cyber attacks," Neurocomputing, 2016. 


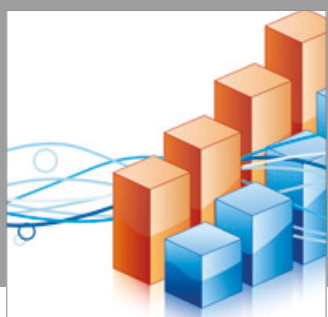

Advances in

Operations Research

vatersals

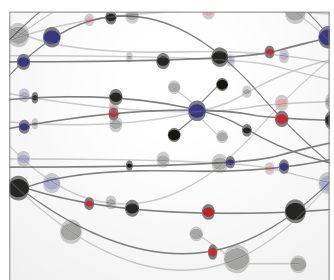

\section{The Scientific} World Journal
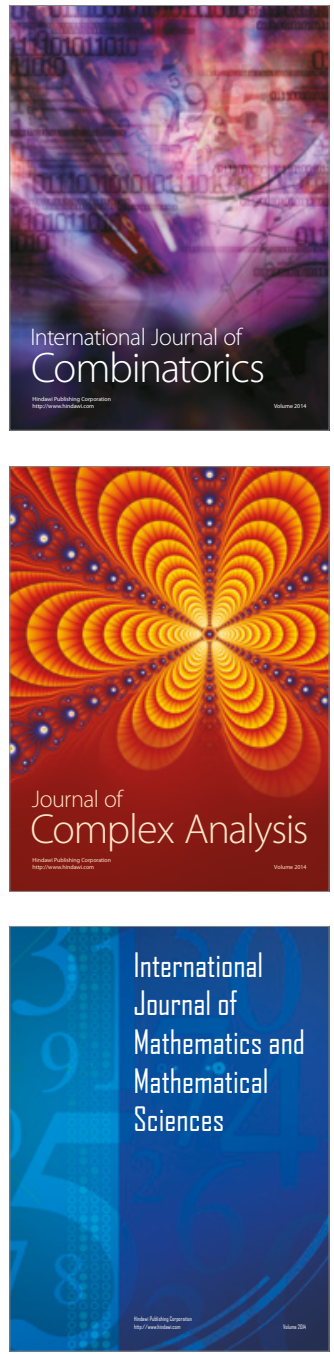
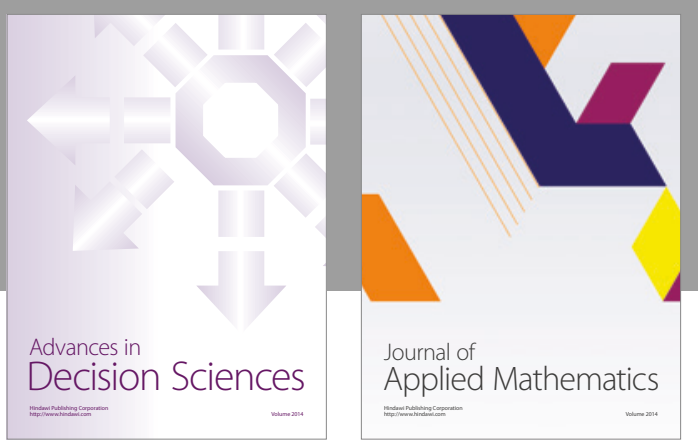

Algebra

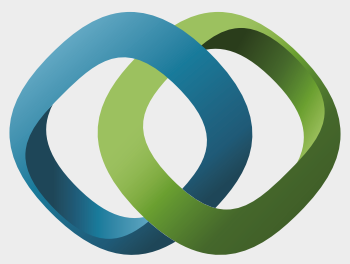

\section{Hindawi}

Submit your manuscripts at

https://www.hindawi.com
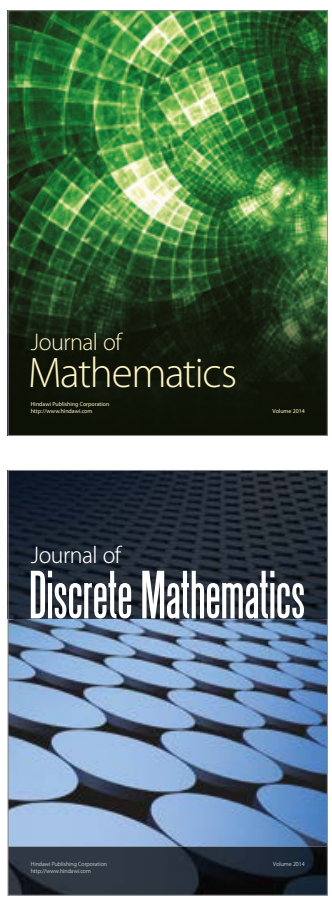

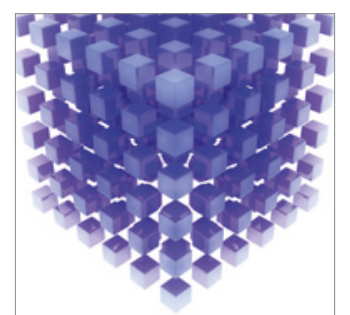

Mathematical Problems in Engineering
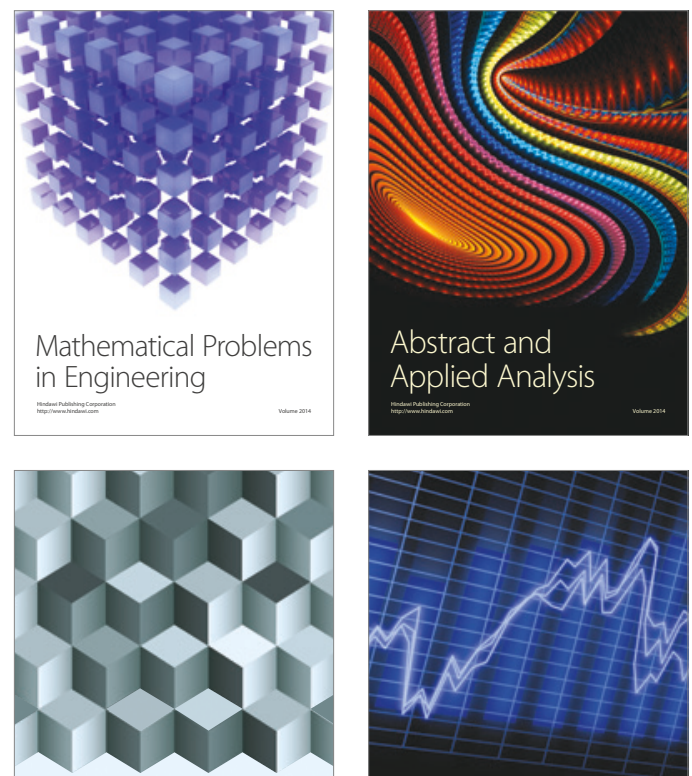

Journal of

Function Spaces

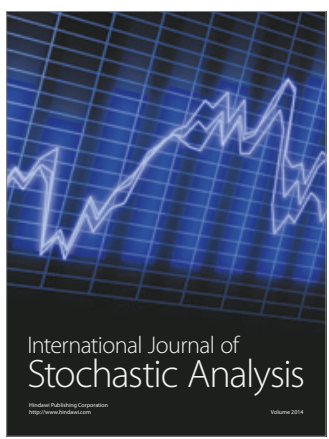

Probability and Statistics
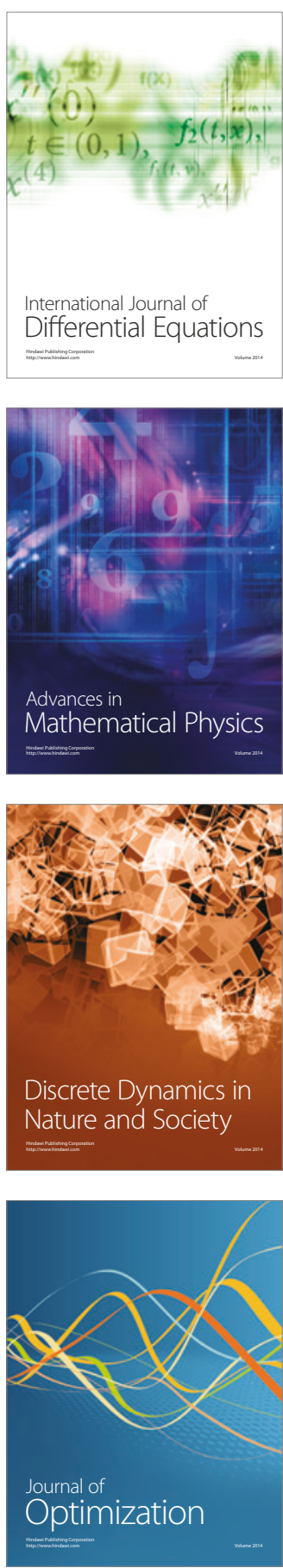